\title{
DAMAGE AND PLASTICITY IN MICROPLANE THEORY
}

\author{
IGNACIO CAROL \\ ETSECCPB-UPC, Barcelona, E-08034 Spain \\ and \\ ZDENĚK P. BAŽANT \\ Northwestern University, Evanston, IL 60208, U.S.A. \\ (Received 10 July 1996 ; in revised form 18 November 1996)
}

\begin{abstract}
The paper deals with the microplane model, in which the stress-strain relations are defined independently on planes of all possible orientations in the microstructure, and the microplane stresses or strains are then constrained kinematically or stalically to the macroscopic stress or strain tensor. The existing formulations of the microplane constitutive model for concrete are mainly based on the kinematic constraint. They have been shown capable of reproducing satisfactorily most experimental results available for concrete specimens, with the advantages of great conceptual simplicity, convenient numerical explicitness, intrinsic induced anisotropy and microcrack openingclosure conditions, etc. However, from the theoretical viewpoint little has been said about how these formulations relate to classical constitutive models of elasto-plasticity or continuum damage mechanics. In this paper, a new aperçu of microplane theory is achieved by systematically introducing damage and plasticity concepts into the microplane framework. New insight is provided on the role played by the split of the normal components, and on the role of the different possible types of micro-macro constraint. Specific formulations are developed and discussed within the new theoretical framework, which can be easily related to von Mises plasticity and to the existing models based on the second and fourth-order damage tensors. (C) 1997 Elsevier Science Ltd.
\end{abstract}

\section{INTRODUCTION}

The idea of developing material laws for the two- or three-dimensional continuum starting from the behavior on a plane of generic orientation is old and has proven very powerful. The classical elasto-plastic failure envelopes such as Tresca and Mohr-Coulomb can be derived from the idea of a limit $\sigma-\tau$ condition for a generic plane (Mohr, 1900). The slip theory of plasticity (Taylor, 1938; Batdorf and Budiansky, 1949) and the viscoplastic multilaminate model for fractured rocks and soils (Zienkiewicz and Pande, 1977; Pande and Sharma, 1983) were also based on similar concepts. The idea was extended by Bažant and $\mathrm{Oh}(1983)$ to strain softening damage and fracturing of materials such as concrete and rock. Because for these materials the original terms "slip theory of plasticity" became obviously unsuitable, the more general term "microplane model", reflecting the fact that this approach merely approximates the micro-macro relations and is not restricted to any particular type of rheologic behavior, was coined.

After successive modifications (Bažant and Gambarova, 1984; Bažant and Oh, 1985) and microplane model reached its classical formulation with kinematic constraint in Bažant and Prat (1988a, b) where it was verified successfully by comparisons with most of the experimental data available for concrete specimens. Microplane formulations have also been developed for anisotropic clays (Bažant and Prat, 1987) and for soils (Prat and Bažant, 1989 ; Prat and Bažant, 1991a, b). Later, the microplane model for concrete was improved to an explicit form (Carol et al., 1992b) which offered much better numerical efficiency with similar data fitting capabilities. Recently, the model was further improved and simplified by introduction of the so-called stress-strain boundaries, and was also generalized to finite strain (Bažant et al., 1996a, b).

All those formulations were based on the assumption of certain stress-strain laws for the microplanes and a micro-macro constraint of the kinematic type. The kinematic 
constraint leads to the expression of the macroscopic stress tensor as an integral over the hemisphere of the normal and shear stresses on each microplane. Damage concepts with the hypothesis of strain equivalence were introduced in Carol $e t$ al. (1991), with the result of a fourth-order damage tensor expressed as the integral of microplane damage variables. This tensor, purely geometric in nature (i.e., totally independent of material rheology), made it possible to combine damage with linear aging viscoelasticity and reproduce longterm failure under sustained loads as given by the Rüsch's curves (Carol and Bažant, 1991; Carol et al., 1992a).

Efficient numerical implementation of the microplane model and its practical application was also studied by Cofer (1992) and Cofer and Kohut (1994). The microplane model from Bažant et al. (1996a, b), was implemented in the large parallel explicit finite element code EPIC at USCE Waterways Experiment Station (WES) to study impact and missile penetration of reinforced concrete walls (M. Adley, private communication, 1996).

In this paper, whose contents were briefly summarized in a recent conference presentation (Carol and Bažant, 1995), a new, extended and more general theoretical framework for microplane formulations is presented. The microplane laws are reformulated in terms of damage and plasticity concepts. This brings about new relations from which macroscopic expressions similar to those in classical plasticity and continuum damage mechanics are obtained. In this way, the microplane formulations can be better understood and compared with the traditional constitutive models. In Section 2, the standard formulation of microplane model with kinematic constraint is briefly reviewed, with a focus on the differences and consequences of introducing the split of normal components into volumetric and deviatoric parts. In Section 3, the conditions for a static micro-macro constraint and a double constraint are explained, and also the expressions of the invariants of stresses and strains and of their deviatoric parts, are developed. The elastic regime of the microplane formulation is revisited in Section 4, with analysis of the differences between the formulation with or without the volumetric-deviatoric split of normal microplane strains and of the identification of initial microplane moduli from the conventional elastic moduli. The condition for a double constraint in the elastic regime is also given. Section 5 focuses on microplane elasto-plasticity, presenting elasto-plastic relations for the microplanes and an integral expression for the macroscopic plastic strains in terms of the microplane plastic strains. An example of a simple model developed in this context is described to demonstrate a full stress explicitness for prescribed strain histories, the hardening due to progressive yielding of the microplanes, and the equivalence to von Mises plasticity for the fully plastic behavior. Damage in the microplanes is introduced in Section 6, with two formulations bascd on the "energy cquivalence approach". The first one, based on the microplane damage variables with the normal-deviatoric split and a fourth-order damage tensor, is similar to the previous formulations in the context of the "strain equivalence approach" (Carol et al., 1991). The second one is based on new developments with microplane damage variables without split and a second-order damage tensor. This formulation is easily related to previous micromechanical and phenomenological developments, and both formulations are specified for elastic and elasto-plastic effective models. Finally, Section 7 presents the main conclusions and some final remarks.

\section{STANDARD MICROPLANE MODEL WITH KINEMATIC CONSTRAINT}

The standard microplane model with kinematic constraint was formulated in detail elsewhere, as already cited. Therefore only the basic assumptions and some essential properties which are relevant to the new developments are outlined in this section.

\subsection{Formulation without volumetric-deviatoric split}

A microplane is a plane of any orientation cutting the material at a given point, and imagined to represent behavior on planes of that orientation in the microstructure. A microplane is defined by its unit normal vector of components $n_{i}$. The normal components of stresses and strains $\sigma_{N}, \varepsilon_{N}$, and the cartesian components $\sigma_{T_{r}}, \varepsilon_{T_{r}}$ of the stress and strain vectors are introduced for each microplane. The kinematic constraint means that the normal 
and shear strain on the microplane, $\varepsilon_{N}, \varepsilon_{T_{r}}$, are equal to the projections of the macroscopic strain tensor $\varepsilon_{i j}$ :

$$
\begin{aligned}
& \varepsilon_{N}=\varepsilon_{i j} n_{i} n_{j} \\
& \varepsilon_{T_{r}}=\varepsilon_{r j} n_{j}-\varepsilon_{N} n_{r}=\left(\delta_{r j}-n_{r} n_{j}\right) n_{k} \varepsilon_{j k}=\frac{1}{2}\left(n_{i} \delta_{j r}+n_{j} \delta_{i r}-2 n_{i} n_{j} n_{r}\right) \varepsilon_{i j}
\end{aligned}
$$

where the Latin lowercase subscripts refer to cartesian coordinates $x_{i}(i=1,2,3)$, and subscript repetition implies summation. The last right-hand expression of (lb), which is symmetric, is obtained by taking advantage of the symmetry of $\varepsilon_{i j}$. Once the kinematic constraint is imposed, equilibrium between the macro- and micro-stresses cannot be satisfied exactly, but it can be satisfied in a weak sense by applying the principle of virtual work (Bažant, 1984). This yields

$$
\sigma_{i j}=\frac{3}{2 \pi} \int_{\Omega} \sigma_{N} n_{i} n_{j} \mathrm{~d} \Omega+\frac{3}{2 \pi} \int_{\Omega} \frac{\sigma_{T r}}{2}\left(n_{i} \delta_{r j}+n_{j} \delta_{r i}\right) \mathrm{d} \Omega
$$

where $\Omega$ is the surface of a unit hemisphere. Equation (2) is based on equality of the virtual work inside a unit sphere and on its surface, which is rigorously justified in Bažant $e t$ al. (1996a).

The formulation of the microplane model without split becomes complete by introducing microplane constitutive laws of the type :

$$
\sigma_{N}=\mathscr{F}_{N}\left(\varepsilon_{N}\right) ; \quad \sigma_{T_{r}}=\mathscr{F}_{T_{r}}\left(\varepsilon_{T}, \varepsilon_{N}\right)
$$

Disregarding the shear contributions, this model corresponds to the original formulation considered for the microplane model with kinematic constraint, which yielded a satisfactory description of the test results for tensile cracking and crack shear (Bažant and Oh, 1983; Bažant and Gambarova, 1984).

An additional property of the kinematically constrained microplane formulation without split, not mentioned in previous publications but useful for our later considerations, is that the strains also satisfy an integral equation analogous to (2):

$$
\varepsilon_{i j}=\frac{3}{2 \pi} \int_{\Omega} \varepsilon_{N} n_{i} n_{j} \mathrm{~d} \Omega+\frac{3}{2 \pi} \int_{\Omega} \frac{\varepsilon_{T_{r}}}{2}\left(n_{i} \delta_{r j}+n_{j} \delta_{r i}\right) \mathrm{d} \Omega
$$

This can be easily verified by substituting into it $\varepsilon_{N}$ and $\varepsilon_{T_{r}}$ from the kinematic constraint $(1 \mathrm{a}, \mathrm{b})$, bringing $\varepsilon_{k l}$ in front of the integrals, and calculating the integrals with the help of relation

$$
\int_{\Omega} n_{i} n_{j} \mathrm{~d} \Omega=\frac{2 \pi}{3} \delta_{i j} .
$$

Equation (4) means that the principle of complementary virtual work, too, is satisfied. It does not mean, however, that a static constraint would be in place (which would imply expressions similar to Equation (1) for $\sigma_{N}$ and $\sigma_{T}$, which are not satisfied for general stressstrain laws).

An alternative way to obtain the weak form of equilibrium (2), apparently not yet mentioned in the literature, is the least-square minimization of the differences between the (given) microplane stresses and the projections on each microplane of the (unknown) stress tensor. The minimization function $\Phi$ and the minimizing conditions are : 


$$
\begin{gathered}
\Phi=\int_{\Omega 2}\left[\left(\sigma_{N}-\sigma_{k l} n_{k} n_{l}\right)^{2}+\left(\sigma_{T_{i}}-\frac{\sigma_{k l}}{2}\left(n_{k} \delta_{r l}+n_{l} \delta_{r k}-2 n_{k} n_{l} n_{r}\right)\right)^{2}\right] \mathrm{d} \Omega \\
\frac{\partial \Phi}{\partial \sigma_{i j}}=-2 \int_{\Omega}\left[\left(\sigma_{N}-\sigma_{k l} n_{k} n_{l}\right) n_{i} n_{j}+\frac{\sigma_{T}}{2}\left(n_{i} \dot{\delta}_{r j}+n_{j} \dot{\delta}_{r i}\right)\right. \\
\left.-\frac{\sigma_{k l}}{4}\left(n_{i} n_{k} \dot{\delta}_{j l}+n_{i} n_{l} \dot{\delta}_{i k}+n_{j} n_{k} \delta_{i l}+n_{j} n_{l} \delta_{i k}-4 n_{i} n_{j} n_{k} n_{l}\right)\right] \mathrm{d} \Omega=0 .
\end{gathered}
$$

The terms with $\sigma_{k l}$ can be grouped together into a separate integral. Then $\sigma_{k l}$ itself can be brought in front of the integral and the integral can be evaluated with the help of (5), which yields eqn (2).

\subsection{Formulation with volumetric-deviatoric split}

Despite satisfactory results in tension and shear, the formulation just described does not capture the volumetric deviatoric interaction observed under compressive stresses for a number of cohesive-frictional materials such as concrete. For that purpose, a split of the normal microplane stresses and strains into volumetric and deviatoric parts, $\sigma_{N}=\sigma_{V}+\sigma_{D}$ and $\varepsilon_{N}=\varepsilon_{1}+\varepsilon_{D}$, was introduced. With the split, the kinematic constraint eqns (1) can be replaced by

$$
\begin{aligned}
& n_{1}=\frac{\delta_{k k}}{3}=\frac{\delta_{i j}}{3} \varepsilon_{i j} \\
& n_{i n}=\varepsilon_{\mathrm{N}}-\varepsilon_{i}=\left(n_{i} n_{i}-\frac{\delta_{i j}}{3}\right) \varepsilon_{i j} \\
& \varepsilon_{T_{r}}=\frac{1}{2}\left(n_{i} \dot{\delta}_{j r}+n_{j} \dot{\delta}_{i r}-2 n_{i} n_{j} n_{r}\right) \varepsilon_{i j} .
\end{aligned}
$$

Note that $\varepsilon_{V}$ and $\sigma_{V}$ are the same for all the microplanes, while $\sigma_{N}, \varepsilon_{N}, \sigma_{D}, \varepsilon_{D}, \sigma_{T}$, and $\varepsilon_{T_{r}}$ change from one microplane to another. Also, note that $\varepsilon_{D}$ can alternatively be considered as the normal component of the projection of the deviatoric strain tensor $e_{i j}=\varepsilon_{i j}-\varepsilon_{l} \delta_{i j}$ onto the microplane, $\varepsilon_{n}=e_{i j} n, n_{j}$. With the split of normal components, the original weak form of equilibrium condition (2) is still valid, but it is advantageous to rewrite it as:

$$
\sigma_{i j}=\sigma_{\nu} \delta_{i j}+\frac{3}{2 \pi} \int_{\Omega} \sigma_{\nu} n_{i} n_{j} \mathrm{~d} \Omega+\frac{3}{2 \pi} \int_{\Omega} \frac{\sigma_{T_{r}}}{2}\left(n_{i} \dot{\delta}_{r j}+n_{j} \delta_{r i}\right) \mathrm{d} \Omega
$$

In this context, the microplane stress strain laws are assumed in the form:

$$
\sigma_{1}=\overline{\mathscr{M}}_{1}\left(\varepsilon_{1}\right) ; \quad \sigma_{1}=\overline{\mathscr{F}}_{n}\left(\varepsilon_{2}\right): \sigma_{T_{1}}=\overline{\mathscr{F}}_{T_{r}}\left(\varepsilon_{T}, \varepsilon_{1}, \varepsilon_{n}\right) .
$$

For the model with split, we again have the property that the strains satisfy an integral expression similar to eqn (9) for stresses:

$$
\dot{c}_{i j}=\varepsilon_{l} \delta_{i j}+\frac{3}{2 \pi} \int_{\Omega \Omega} \varepsilon_{n} n_{i} n_{j} \mathrm{~d} \Omega+\frac{3}{2 \pi} \int_{\Omega 2} \frac{\varepsilon_{T}}{2}\left(n_{i} \delta_{r i}+n_{j} \delta_{r i}\right) \mathrm{d} \Omega
$$

This relation can be verified by substituting $(8 \mathrm{a}, \mathrm{b}, \mathrm{c})$ and evaluating the integrals. It does not imply a static constraint between the microplane and macroscopic stresses. 


\subsection{Discussion}

In the initial microplane formulation without the volumetric-deviatoric split, the normal microplane stress $\sigma_{N}$ was exclusively a function of the normal strain on the same microplane, $\varepsilon_{N}$; eqn (3a). In the formulation with the split, one can sum (10a) and (10b) to obtain $\sigma_{N}=\sigma_{V}+\sigma_{D}=\mathscr{F}_{V}\left(\varepsilon_{V}\right)+\mathscr{F}_{D}\left(\varepsilon_{D}\right)=\mathscr{F}_{N}^{\prime}\left(\varepsilon_{N}, \varepsilon_{V}\right)$; i.e., $\sigma_{N}$ becomes also a function of the strains in other directions (in this case, of $\varepsilon_{V}$ which is the average of the normal strains on all microplanes). This coupling of the normal behavior of the microplanes, rather than the split itself, seems to be the essential requirement for the model to capture the type of macroscopic volumetric-deviatoric interaction observed in frictional materials. This argument is verified by the formulation of Hasegawa and Bažant (1993a, b), which did not use the split but includes certain dependence of $\sigma_{N}$ on the lateral stresses and strains $\sigma_{L}$ and $\varepsilon_{L}$ (i.e., the normal stresses and strains on planes perpendicular to $n_{i}$ ). However, that alternative and others already mentioned turn out to be more complex and to require a higher number of parameters than the standard formulation with the split of normal components. In addition, the normal-volumetric cross-effects need not only be introduced for the nonlinear range, but they also do for the linear elastic behavior if the full range of Poisson ratios is to be reproduced. This is naturally achieved by the standard formulation with the split (see Section 4) but remains unattained by other approaches such as Hasegawa and Bažant's (1993a, b).

The variables and relations involved in the standard kinematically constrained microplane model with the volumetric-deviatoric split are shown in Fig. 1. The arrows on the solid lines indicate the flow of explicit relations from the strain tensor to the strains on any given microplane (kinematic constraint), then to the stresses on the same microplane (microplane stress-strain laws), and finally to the macroscopic stress, by integration over all possible microplanes on the hemisphere (eqns 2 or 9 ). The integrals over the hemisphere are performed numerically by summation over a fixed number of "sample" orientations (normally 21,25 or 28 for three-dimensional calculations). These are the discrete orientations of the microplanes for which all the variables are calculated and for which the history variables for the material laws are stored and updated. The details of the stressstrain laws, integration rules and results obtained for a variety of tests of concrete can be found in Bažant and Prat (1988b), Carol et al. (1992b) and Bažant et al. (1996a, b). The kinematically constrained microplane model has the advantage that all the calculations of the strain-to-stress type (which is the way usually needed in computer applications) may be carried out explicitly, i.e., without the need for any step-by-step or iterative integration procedure in the loading steps.

Although, in most loading situations, Bažant and Prat's (1988) model with the volumetric-deviatoric split performs satisfactorily, it has recently been found that, under uniaxial loading with large postpeak tensile strains (implying tensile cracking), the intrinsic

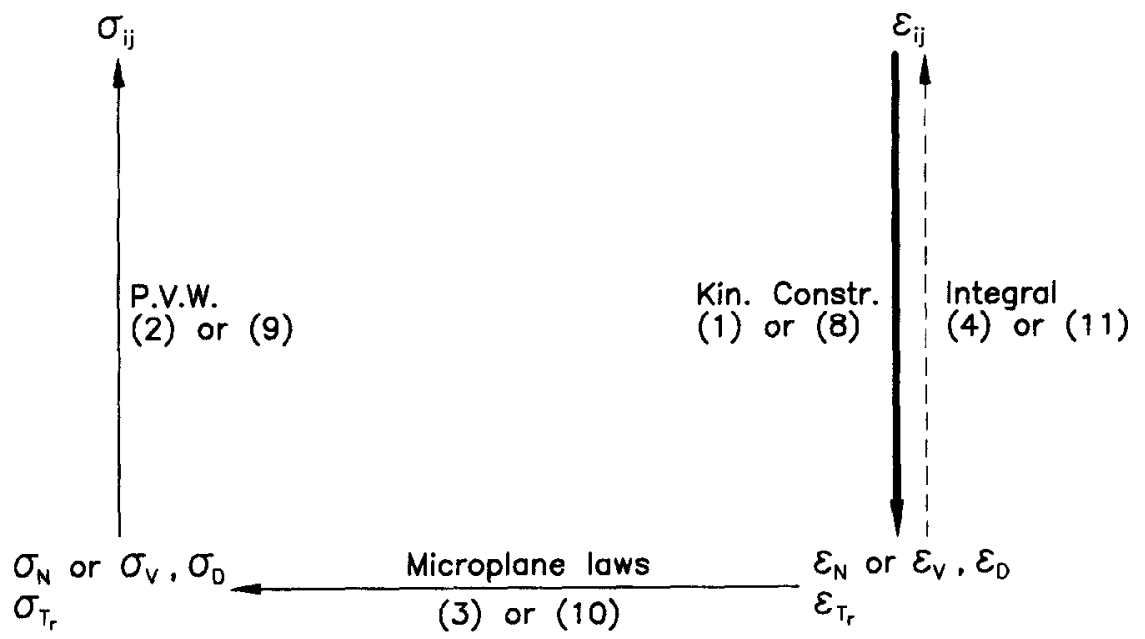

Fig. 1. Variables and relations in the standard microplane model with kinematic constraint. 
normal-deviatoric coupling causes unrealistic volumetric expansion which cannot be eliminated (Bažant et al., 1994; Bažant et al., 1996). Therefore, under these particular conditions, the original Bažant and Oh (1993) formulation without split would in fact be preferable. As a remedy, Bažant et al. $(1994,1996 a$, b) proposed a new model with socalled stress-strain boundaries formulated in such a way that for all compression response and for tensile elastic loading there is a split, while for tensile strain-softening there is no split. This solves the problem at the expenses of a certain number of "IF" conditions which necessarily cause some discontinuities of the slope of the stress-strain response curves because the number of the microplanes involved in the numerical implementation is finite. The introduction of damage and plasticity concepts into a unified microplane framework, as proposed in this paper, makes it possible to envisage an alternative, more natural way to combine the models with and without the split, and obtained a smooth continuous transition between both extreme situations depending on the loading conditions. This is explained in more detail in Section 6.

\section{STATIC OR DOUBLE CONSTRAINTS, AND STRESS OR STRAIN INVARIANTS}

It is of interest to consider also the static constraint, which is dual to the kinematic constraint considered in the previous section. The static constraint means that the microplane stresses $\sigma_{N}, \sigma_{V}, \sigma_{D}$ and $\sigma_{T_{r}}$ are the projections of the stress tensor $\sigma_{i j}$. For a formulation without the volumetric-deviatoric split, this means that

$$
\begin{aligned}
& \sigma_{N}=n_{i} n_{j} \sigma_{i j} \\
& \sigma_{T_{r}}=\frac{1}{2}\left(n_{i} \delta_{r j}+n_{j} \delta_{r i}-2 n_{i} n_{j} n_{r}\right) \sigma_{i j} .
\end{aligned}
$$

For a formulation with split, the static constraint would read:

$$
\begin{aligned}
& \sigma_{V}=\frac{\dot{\partial}_{i j}}{3} \sigma_{i j} \\
& \sigma_{D}=\left(n_{i} n_{j}-\frac{\delta_{i j}}{3}\right) \sigma_{i j} \\
& \sigma_{r_{r}}=\frac{1}{2}\left(n_{i} \delta_{r j}+n_{j} \delta_{r i}-2 n_{i} n_{j} n_{r}\right) \sigma_{i j}
\end{aligned}
$$

where (13a) and (13b) replace (12a). In both cases, the integral expressions for stresses (2) and strains (4) hold (eqn 2 can be obtained from the principle of virtual work, and eqn 4 can be verified by substitution of eqns 13). In the case with the split, (2) and (4) can also be written as in (9) and (11).

With the previous definitions, it is immediately obvious that one can formulate dual models based on the static constraint exclusively. It has been verified, however, that such models do not exhibit a stable behavior in the softening regime. Therefore, such models are limited to the hardening part of the strain-stress behavior, which is not sufficient to handle damage. Nevertheless, the static constraint is useful in some instances, as it will become apparent later. In particular, one can consider models with a double constraint, i.e., models that satisfy the static constraint (12) or (13) and the kinematic constraint (1) or (8) simultaneously, and also satisfy the two integral eqns (2) and (4) which, in the case of split, can also be written as (9) and (11).

To clarify the relation of microplane formulations to traditional constitutive models based on tensors and invariants, it is useful to obtain expressions for the invariants of the macroscopic stress and strain tensors or their deviatoric parts in terms of the microplane variables. The first stress invariant $I_{1}$ is obtained directly from integral eqns (2) or (9) : 


$$
I_{1}=\sigma_{k k}=\frac{3}{2 \pi} \int_{\Omega} \sigma_{N} \mathrm{~d} \Omega \quad \text { or } \quad I_{1}=3 \sigma_{V}+\frac{3}{2 \pi} \int_{\Omega} \sigma_{D} \mathrm{~d} \Omega .
$$

The deviatoric stresses then follow from (9) and (14b):

$$
\begin{aligned}
s_{i j} & =\sigma_{i j}-\frac{\sigma_{k k}}{3} \delta_{i j} \\
& =\frac{3}{2 \pi} \int_{\Omega} \sigma_{D}\left(n_{i} n_{j}-\frac{\delta_{i j}}{3}\right) \mathrm{d} \Omega+\frac{3}{2 \pi} \int_{\Omega} \frac{\sigma_{T_{r}}}{2}\left(n_{i} \delta_{r j}+n_{j} \delta_{r i}-2 n_{i} n_{j} n_{r}\right) \mathrm{d} \Omega .
\end{aligned}
$$

Note that the last term $-2 n_{i} n_{j} n_{r}$ multiplied with $\sigma_{T_{r}}$ yields zero. That term was omitted in the original integral expressions (2) and (9), but will be needed later. Similarly, for the first invariant of the strain tensor,

$$
I_{1}=\varepsilon_{k k}=\frac{3}{2 \pi} \int_{\Omega} \varepsilon_{N} \mathrm{~d} \Omega \quad \text { or } \quad I_{1}=3 \varepsilon_{V}+\frac{3}{2 \pi} \int_{\Omega} \varepsilon_{D} \mathrm{~d} \Omega
$$

The deviatoric strains then follow from (11) and (16b):

$$
\begin{aligned}
e_{i j} & =\varepsilon_{i j}-\frac{\varepsilon_{k k}}{3} \delta_{i j} \\
& =\frac{3}{2 \pi} \int_{\Omega} \varepsilon_{D}\left(n_{i} n_{j}-\frac{\delta_{i j}}{3}\right) \mathrm{d} \Omega+\frac{3}{2 \pi} \int_{\Omega} \frac{\varepsilon_{T_{r}}}{2}\left(n_{i} \delta_{r j}+n_{j} \delta_{r i}-2 n_{i} n_{j} n_{r}\right) \mathrm{d} \Omega .
\end{aligned}
$$

Both foregoing expressions for the first stress and strain invariants are valid for either kinematic or static constraint.

By contrast, the expressions for the second and third invariants are valid only for specific kinds of constraint. The second and third invariants of stress require the static constraint, while those of strain require the kinematic constraint. If the double constraint is in place, all the expressions which follow apply. It is convenient to consider the following definitions of quadratic invariants of the total and deviatoric stress and strain tensors:

$$
I_{2}^{*}=\frac{1}{2} \sigma_{i j} \sigma_{i j} \quad J_{2}=\frac{1}{2} s_{i j} s_{i j} \quad I_{2}^{*}=\frac{1}{2} \varepsilon_{i j} \varepsilon_{i j} \quad J_{2}=\frac{1}{2} e_{i j} e_{i j} .
$$

Here $J_{2}$ and $\bar{J}_{2}$ are the second invariants of the deviators, while the second invariants of stress and strain are $I_{2}=I_{2}^{*}-I_{1}^{2} / 2$ and $I_{2}=\Gamma_{2}^{*}-\Gamma_{1}^{2} / 2$. For $I_{2}^{*}$, the integral eqn (2) for $\sigma_{i j}$ without the split is multiplied by the factor $\sigma_{i j} / 2$, which can go as a constant into the integrals since it does not depend on the particular orientation of the microplane

$$
2 I_{2}^{*}=\frac{3}{2 \pi} \int_{\Omega} \sigma_{N} n_{i} n_{j} \sigma_{i j} \mathrm{~d} \Omega+\frac{3}{2 \pi} \int_{\Omega} \sigma_{T_{r}} \frac{1}{2}\left(n_{i} \delta_{j r}+n_{j} \delta_{i r}-2 n_{i} n_{j} n_{r}\right) \sigma_{i j} \mathrm{~d} \Omega .
$$

Now, if the static constraint is satisfied, one can identify the products leading to $\sigma_{N}$ and $\sigma_{T_{r}}(12)$, group the two integrals, and obtain:

$$
2 I_{2}^{*}=\frac{3}{2 \pi} \int_{\Omega}\left(\sigma_{N}^{2}+\sigma_{T}^{2}\right) \mathrm{d} \Omega
$$

where $\sigma_{T}^{2}=\sigma_{T_{r}} \sigma_{T_{r}}$. Similarly, one can use the integral expression for $s_{i j}(15)$, multiply it by $s_{i j} / 2$ (which goes into the integrals), identify $\sigma_{D}$ and $\sigma_{T_{r}}$ from the static constraint equations, and obtain 


$$
2 J_{2}=\frac{3}{2 \pi} \int_{\Omega}\left(\sigma_{D}^{2}+\sigma_{T}^{2}\right) \mathrm{d} \Omega \text {. }
$$

Analogous developments for strains can be followed if the kinematic constraint is satisfied, leading to :

$$
\begin{aligned}
& 2 \bar{I}_{2}^{*}=\frac{3}{2 \pi} \int_{\Omega}\left(\varepsilon_{N}^{2}+\varepsilon_{T}^{2}\right) \mathrm{d} \Omega \\
& 2 \bar{J}_{2}=\frac{3}{2 \pi} \int_{\Omega}\left(\varepsilon_{D}^{2}+\varepsilon_{T}^{2}\right) \mathrm{d} \Omega .
\end{aligned}
$$

It is also convenient to consider the cubic invariants of stress and strain, defined as :

$$
I_{3}^{*}=\frac{1}{3} \sigma_{i j} \sigma_{j k} \sigma_{k i} \quad J_{3}=\frac{1}{3} s_{i j} s_{j k} s_{k i} \quad \bar{I}_{3}^{*}=\frac{1}{3} \varepsilon_{i j} \varepsilon_{j k} \varepsilon_{k i} \quad \bar{J}_{3}=\frac{1}{3} e_{i j} e_{j k} e_{k i} . \quad(24 \mathrm{a}, \mathrm{b}, \mathrm{c}, \mathrm{d})
$$

Here $J_{3}$ and $\bar{J}_{3}$ are the third invariants of the deviators. The third invariants of stress and strain are $I_{3}=I_{3}^{*}-I_{1} I_{2}-I_{1}^{3} / 3$ and $\bar{I}_{3}=I_{3}^{*}-I_{1} I_{2}-\Gamma_{1} / 3$. To relate $I_{3}^{*}$ to the microplane stresses, one can start from any integral of the third-power terms of $\sigma_{N}, \sigma_{T_{r}}$ or both. By definition, any scalar integral of microplane variables over the hemisphere must be invariant with rotations, and therefore equal to some combination of $I_{1}, I_{2}$ and $I_{3}$. In particular, one can consider the third-power integrand $\sigma_{N}\left(\sigma_{N}^{2}+\sigma_{T}^{2}\right)$, which, after replacing the static constraint (12) and moving the stress tensors in front of the integral, yields

$$
\int_{\Omega} \sigma_{N}\left(\sigma_{N}^{2}+\sigma_{T}^{2}\right) \mathrm{d} \Omega=\sigma_{i j} \sigma_{k l} \sigma_{p q} \int_{\Omega} \frac{1}{4} n_{i} n_{j}\left(n_{k} n_{p} \delta_{l_{i}}+n_{k} n_{q} \delta_{l p}+n_{l} n_{p} \delta_{k q}+n_{l} n_{q} \delta_{k p}\right) \mathrm{d} \Omega
$$

The integral can be evaluated with the help of the following equation (Lubarda and Krajcinovic, 1993)

$$
\int_{\Omega} n_{i} n_{j} n_{k} n_{l} \mathrm{~d} \Omega=\frac{2 \pi}{15}\left(\delta_{i j} \delta_{k l}+\delta_{i k} \delta_{j l}+\delta_{i l} \delta_{j k}\right)
$$

After calculating the products of the Kronecker deltas with the stress tensors and identifying the invariants, one finally obtains:

$$
3 I_{3}^{*}=\frac{5}{2} \frac{3}{2 \pi} \int_{\Omega} \sigma_{N}\left(\sigma_{N}^{2}+\sigma_{T}^{2}\right) \mathrm{d} \Omega-I_{1} I_{2}^{*}
$$

An analogous development with $\sigma_{D}$ and $s_{i j}$ instead of $\sigma_{N}$ and $\sigma_{i j}$, and consideration of the fact that, by definition, $J_{1}=s_{i i}=0$, leads to the equation

$$
3 J_{3}=\frac{5}{2} \frac{3}{2 \pi} \int_{\Omega} \sigma_{D}\left(\sigma_{D}^{2}+\sigma_{T}^{2}\right) \mathrm{d} \Omega
$$

Similar equations hold for the third invariants of strain, if the kinematic constraint is in place: 


$$
\begin{gathered}
3 \bar{I}_{3}^{*}=\frac{5}{2} \frac{3}{2 \pi} \int_{\Omega} \varepsilon_{N}\left(\varepsilon_{N}^{2}+\varepsilon_{T}^{2}\right) \mathrm{d} \Omega-\bar{I}_{1} \bar{I}_{2}^{*} \\
3 \bar{J}_{3}=\frac{5}{2} \frac{3}{2 \pi} \int_{\Omega} \varepsilon_{D}\left(\varepsilon_{D}^{2}+\varepsilon_{T}^{2}\right) \mathrm{d} \Omega .
\end{gathered}
$$

\section{ELASTIC BEHAVIOR}

\subsection{Formulation without split}

In the elastic regime, the microplane laws for the model without the split can be simply written as

$$
\sigma_{N}=E_{N}^{0} \varepsilon_{N} ; \quad \sigma_{T_{r}}=E_{T}^{0} \varepsilon_{T_{r}}
$$

By substituting these and the kinematic constraint eqns (1) into (2), moving the strain tensor in front of the integral, evaluating the integral with the help of (26) and comparing the result to the standard isotropic linear elastic stiffness tensor, one can obtain the equivalence conditions :

$$
E_{N}^{0}=\frac{E}{1-2 v}, \quad E_{T}^{0}=\frac{E(1-4 v)}{(1+v)(1-2 v)}
$$

where $E^{0}$ and $v$ are the Young's modulus and Poisson ratio.

\subsection{Model with split}

For the model with split, the microplane laws in the elastic regime can be written as

$$
\sigma_{V}=E_{V}^{0} \varepsilon_{V} ; \quad \sigma_{D}=E_{D}^{0} \varepsilon_{D} ; \quad \sigma_{T_{r}}=E_{T}^{0} \varepsilon_{T_{r}} .
$$

By substituting these and the kinematic constraint (8) into (9), moving the strain tensor in front of the integral, evaluating the integral with the help of (26) and comparing the result to the standard isotropic linear elastic stiffness tensor, one can obtain the equivalence conditions :

$$
E_{V}^{0}=\frac{E}{1-2 v} ; \quad E_{D}^{0}=\frac{5 E}{1+v} \frac{1}{2+3 \chi_{0}} ; \quad E_{T}^{0}=\chi_{0} E_{D}^{0}
$$

These are the same relations given by Bažant and Prat (1988a) in terms of parameter $n_{0}=E_{D}^{0} / E_{V}^{0}=5(1-2 v) /\left[(1+v)\left(2+3 \chi_{0}\right)\right]$ instead of $\chi_{0}$. Parameter $\chi_{0}$ can be considered as a third elastic constant additional to $E^{0}$ and $v$, which may be chosen arbitrarily for convenience, since it has no significant influence on the data fitting capabilities. Note that the case with $\chi_{0}=(1-4 v) /(1+v)$, which corresponds to $E_{D}^{0}=E_{V}^{0}$, yields the case with no volumetric-deviatoric split.

The Lamé elastic constants are $\lambda^{0}=2 v E^{0} /[(1+v)(1-2 v)]$ and $\mu^{0}=E^{0} /[2(1+v)]$, which furnishes

$$
\lambda^{0}=\frac{1}{15}\left(5 E_{V}^{0}-2 E_{D}^{0}-3 E_{T}^{0}\right), \quad \mu^{0}=\frac{1}{10}\left(2 E_{D}^{0}+3 E_{T}^{0}\right) .
$$

\subsection{Discussion and double constraint}

The first consequence of eqns (32) is that, in the elastic range, the model without the volumetric-deviatoric split can represent Poisson ratios only between -1 and 0.25 , because larger values (between 0.25 and 0.5 ) would generate negative $E_{N}^{0}$ (Bažant and Prat, 1988a). The case with $v=0.25$ yields precisely $E_{T}^{0}=0$, which actually corresponds to the first 
version of the microplane model (Bažant and Oh, 1983; Bažant and Gambarova, 1984), which used only the normal stress and strain components, exhibited a fixed value of $v$, and was coupled in series with an additional elastic element to obtain any desired $v$. By contrast, the complete range of Poisson ratios between -1 and 0.5 can be covered (with positive microplane moduli) in the model with split, if the value of $\chi_{0}$ is chosen within a certain range depending on $v$.

When first proposed, $\chi_{0}$ (or $\eta_{0}$ ) was considered free to choose (Bažant and Prat, 1988a). Later, however, it was noted that, in order to obtain certain convenient features such as the existence of a rheology-free damage tensor (based on the strain equivalence approach, see Carol et al., 1991), $\eta_{0}$ had to be taken as $\eta_{0}=(1-2 v) /(1+v)$, which is the same as

$$
\chi_{0}=1 .
$$

The foregoing value for $\chi_{0}$ coincides with the condition for the elastic microplane formulation to satisfy the double constraint, i.e., the kinematic and static constraints simultaneously (Carol and Bažant, 1995). The proof is as follows : for the elastic microplane formulation with the split and the unrestricted moduli $E_{V}^{0}, E_{D}^{0}$ and $E_{T}^{0}$, the stress tensor is related to the strain tensor through the standard Lamé elastic equations in which the Lamé constants are given by (35):

$$
\sigma_{i j}=\frac{1}{15}\left(5 E_{V}^{0}-2 E_{D}^{0}-3 E_{T}^{0}\right) \varepsilon_{k k} \delta_{i j}+\frac{1}{5}\left(2 E_{D}^{0}+3_{T}^{0}\right) \varepsilon_{i j}
$$

The volumetric, normal deviatoric and shear projections $\bar{\sigma}_{V}, \bar{\sigma}_{D}$ and $\bar{\sigma}_{T_{r}}$ of $\sigma_{i j}$ can then be obtained as

$$
\begin{aligned}
\bar{\sigma}_{V} & =E_{V}^{0} \frac{\varepsilon_{k k}}{3} \\
\bar{\sigma}_{D} & =\frac{1}{5}\left(2 E_{D}^{0}+3 E_{T}^{0}\right)\left(n_{i} n_{j}-\frac{\delta_{i j}}{3}\right) \varepsilon_{i j} \\
\bar{\sigma}_{T_{r}} & =\frac{1}{5}\left(2 E_{D}^{00}+3 E_{T}^{00}\right) \frac{1}{2}\left(n_{i} \delta_{j r}+n_{j} \delta_{i r}-2 n_{i} n_{j} n_{r}\right) \varepsilon_{i j} .
\end{aligned}
$$

Now, if the double constraint is to be satisfied, the replacement of the projections of the strain tensor according to the kinematic constraint (8) shows that $\bar{\sigma}_{V}, \bar{\sigma}_{D}$ and $\bar{\sigma}_{T \text {, }}$ should be equal to $\sigma_{V}, \sigma_{D}$ and $\sigma_{T}$, respectively, as given in (33). This means that $\left(2 E_{D}^{0}+3 E_{T}^{0}\right) / 5$ must simultaneously be equal to $E_{D}^{0}$ and to $E_{T}^{0}$, which can be possible only if $E_{D}^{0}=E_{T}^{0}$. From this condition and eqns (34), one finally obtains relation (36); Q.E.D.

The double constraint turns out to be a convenient feature of the elastic model and the requirement for some of the developments presented in the following sections. With it, the elastic microplane stiffnesses become

$$
E_{V}^{0}=\frac{E}{1-2 v} ; \quad E_{D}^{0}=\frac{E}{1+v} ; \quad E_{T}^{0}=E_{D}^{0}
$$

It must be noted that a consistent double constraint exists only for the elastic model with volumetric-deviatoric split of normal microplane components. It is a simple exercise to verify that, in the model without the split, the double constraint requires the Lamé elastic constant $\lambda^{0}=0$, which corresponds to an unrealistic linear elastic stiffness tensor proportional to the fourth-order identity tensor $E_{i j k l}^{0}=\mu^{0}\left(\delta_{i k} \delta_{j l}+\delta_{i l} \delta_{j k}\right)$. Together with the foregoing arguments on the range of Poisson ratio represented by the model, this indicates that the linear elastic backbone of any nonlinear microplane formulation should be the model with volumetric-deviatoric split. 
Another way to establish the relation between the double constraint and the volumetric-deviatoric split in the elastic range is to consider the products of the elastic relation $\sigma_{i j}=2 \mu \varepsilon_{i j}+\lambda \varepsilon_{k k} \delta_{i j}$ with each of the three factors $\delta_{i j}, n_{i} n_{j}$ and $\left(n_{i} \delta_{r j}+n_{j} \delta_{r i}-2 n_{i} n_{j} n_{r}\right) / 2$. Assuming the double constraint, in the first and third cases, one obtains (33a) with $E_{V}^{0}$ given by (39a), and (33c) with $E_{T}^{0}$ given by $(39 b)$, respectively. With the double constraint and the second factor $n_{i} n_{j}$, one obtains the relation $\sigma_{N}=2 \mu \varepsilon_{N}+3 \lambda \varepsilon_{V}$, which shows the need for cross-influence between volumetric and normal components on each microplane. Introducing the volumetric-deviatoric split, one can substitute $\varepsilon_{N}=\varepsilon_{V}+\varepsilon_{D}$ to obtain $\sigma_{N}=2 \mu\left(\varepsilon_{V}+\varepsilon_{D}\right)+3 \lambda \varepsilon_{V}=E_{V}^{0} \varepsilon_{V}+E_{D}^{0} \varepsilon_{D}$, with microplane moduli given by (39). Further, from the result of multiplication with $\delta_{i j}$, one can identify the first term on the right-hand side of the previous equation as $\sigma_{\nu}$, and thus define the second term as the difference $\sigma_{D}=\sigma_{N}-\sigma_{V}$. In this way, it is shown that classical linear elasticity is equivalent to an elastic microplane model with volumetric-deviatoric split and double constraint.

\section{MICROPLANE ELASTO-PLASTICITY}

5.1. Assumptions and integral equation for plastic strain

Elasto-plasticity can be introduced into the microplane formulation under the classical assumptions that the strain is a sum of elastic and plastic parts (denoted by superscripts $e$ and $p$ ) and that the elastic strains are related to the stresses according to the initial elastic moduli. For the model with volumetric-deviatoric split, this reads

$$
\begin{gathered}
\varepsilon_{V}=\varepsilon_{V}^{e}+\varepsilon_{V}^{p} ; \quad \varepsilon_{V}^{e}=\frac{\sigma_{V}}{E_{V}^{0}} \\
\varepsilon_{D}=\varepsilon_{D}^{e}+\varepsilon_{D}^{p} ; \quad \varepsilon_{D}^{e}=\frac{\sigma_{D}}{E_{D}^{0}} \\
\varepsilon_{T_{r}}=\varepsilon_{T_{r}}^{e}+\varepsilon_{T_{r}}^{p} ; \quad \varepsilon_{T_{r}}^{e}=\frac{\sigma_{T_{r}}}{E_{T}^{0}}
\end{gathered}
$$

Therefore,

$$
\sigma_{V}=E_{V}^{0}\left(\varepsilon_{V}-\varepsilon_{V}^{p}\right) ; \quad \sigma_{D}=E_{D}^{0}\left(\varepsilon_{D}-\varepsilon_{D}^{p}\right) ; \quad \sigma_{T_{r}}=E_{T}^{0}\left(\varepsilon_{T_{r}}-\varepsilon_{T_{r}}^{0}\right)
$$

On the macroscopic level, the classical elasto-plastic relation reads

$$
\sigma_{i j}=E_{i j k l}^{0} \varepsilon_{k l}^{e} ; \quad \varepsilon_{i j}^{e}=\varepsilon_{i j}-\varepsilon_{i j}^{p}
$$

where $\varepsilon_{i j}^{p}$ is to be related to the microplane plastic strains $\varepsilon_{V}^{p}, \varepsilon_{D}^{D}$ and $\varepsilon_{T_{r}}^{p}$

It is assumed first that moduli $E_{N}^{0}, E_{D}^{0}$ and $E_{T}^{0}$ satisfy relations (39), i.e., the elastic part of the model exhibits the double constraint. This means that the stresses satisfy relations (13) and (9) and the elastic strains satisfy

$$
\begin{aligned}
& \varepsilon_{V}^{e}=\frac{\delta_{i j}}{3} \varepsilon_{i j}^{e} \\
& \varepsilon_{D}^{e}=\left(n_{i} n_{j}-\frac{\delta_{i j}}{3}\right) \varepsilon_{i j}^{e} \\
& \varepsilon_{T_{r}}^{e}=\frac{1}{2}\left(n_{i} \delta_{r j}+n_{j} \delta_{r i}-2 n_{i} n_{j} n_{r}\right) \varepsilon_{i j}^{e}
\end{aligned}
$$




$$
\varepsilon_{i j}^{e}=\varepsilon_{V}^{e} \delta_{i j}+\frac{3}{2 \pi} \int_{\Omega} \varepsilon_{D}^{c} n_{i} n_{j} \mathrm{~d} \Omega+\frac{3}{2 \pi} \int_{\Omega} \frac{\varepsilon_{T_{r}}^{c}}{2}\left(n_{i} \delta_{r j}+n_{j} \delta_{r i}\right) \mathrm{d} \Omega .
$$

Considering now the elasto-plastic model, the stresses are the same as for the elastic model, i.e., they satisfy the static constraint (13) and the integral equation (9). This means that the overall elasto-plastic model exhibits the static constraint. Therefore, by applying the principle of virtual work, the same integral equation for total strains as (11) is also satisfied.

Isolating $\varepsilon_{i j}^{p}$ from (44b), substituting the integral expressions (11) for $\varepsilon_{i j}$ and (46) for $\varepsilon_{i j}^{i}$, and using the microplane strain decomposition (40a)-(42a), one obtains the following equation:

$$
\varepsilon_{i j}^{p}=\varepsilon_{V}^{p} \delta_{i j}+\frac{3}{2 \pi} \int_{\Omega} \varepsilon_{D}^{p} n_{i} n_{j} \mathrm{~d} \Omega+\frac{3}{2 \pi} \int_{\Omega} \frac{\varepsilon_{T_{i}}^{p}}{2}\left(n_{i} \dot{\delta}_{t j}+n_{j} \delta_{r i}\right) \mathrm{d} \Omega
$$

This means that the macroscopic plastic strains are obtained as the integral of the plastic microplane strains over all possible orientations. This microplane formulation is conceptually similar to Batdorf and Budianski's (1949) statically constrained "slip theory of plasticity", although those authors considered only shear (slip) microplane plastic strains, which were obtained as the results of another integral over all possible directions on the slip plane.

\subsection{Microplane elasto-plasticity with double constraint}

In general, microplane elasto-plasticity satisfies only the static constraint, and the total microplane strains $\varepsilon_{k}, \varepsilon_{D}$ and $\varepsilon_{T}$, are not necessarily the projections of the macroscopic total strain tensor $\varepsilon_{i j}$. This can be understood from the basic eqns (40a)-(42a). Indeed, with general material laws the plastic components $\varepsilon_{l}^{p}, \varepsilon_{i}^{p}$ and $\varepsilon_{T_{r}}^{p}$ will not be the projections of a tensor; therefore, even if the elastic components $\varepsilon_{V}^{v}, \varepsilon_{D}^{c}$ and $\varepsilon_{T}^{e}$ are, the total strains equal to the sum of the two components will not be the projections of a tensor either.

It is possible, however, to conceive a microplane elasto-plastic formulation with particular material laws such that the kinematic constraint exists and, therefore, the double constraint does, too. This is actually an important type of models, since the double constraint is required for a model to act as the effective part of a microplane damage formulation, as will be explained later. The particular model described in the next section is of this type. In the case that the kinematic constraint is also in place, eqns (8) are satisfied for the total strains and, by subtracting their counterparts for the elastic components (45), one finds that the plastic components also satisfy their own similar constraint equations:

$$
\begin{aligned}
& \varepsilon_{i}^{p}=\frac{\delta_{i j}}{3} \varepsilon_{i j}^{p} \\
& \varepsilon_{D}^{p}=\left(n_{i} n_{j}-\frac{\delta_{i j}}{3}\right) \varepsilon_{i j}^{p} \\
& \varepsilon_{T_{r}}^{\prime \prime}=\frac{1}{2}\left(n_{i} \dot{\delta}_{r j}+n_{j} \dot{\delta}_{r i}-2 n_{i} n_{j} n_{r}\right) \varepsilon_{i j}^{p} .
\end{aligned}
$$

\subsection{Example of a pressure-insensitive microplane elasto-plastic model}

5.3.1. Yield surface. Consider the following fixed circular yield surface in terms of microplane stresses $\sigma_{D}$ and $\sigma_{r}=\sqrt{\sigma_{2,} \sigma_{T}}$, and its alternative expression in terms of $\sigma_{N}, \sigma_{V}$ :

$$
F\left(\sigma_{l}, \sigma_{D}, \sigma_{T}\right)=\sigma_{D}^{2}+\sigma_{T}^{2}-R^{2}=\left(\sigma_{N}-\sigma_{V}\right)^{2}+\sigma_{T}^{2}-R^{2}=0
$$

$\sigma_{N}$ and $\sigma_{T}$ are the coordinates of Mohr circles. In these coordinates, the yield surface is 
represented by a circle of radius $R$, centered on the horizontal axis at a distance $\sigma_{V}$ from the origin. It must be understood that this circle represents a yield condition of perfect plasticity which applies independently for each possible microplane orientation. When macroscopic strains are applied, the stresses on various microplanes increase at different rates, until the yield condition is first reached on one microplane. If, after that, the macroscopic strains continue increasing proportionally, the yielding spreads to microplanes of other orientations, until a final or saturated state is reached. At that moment, all the microplane stresses are on their corresponding yield surface.

It is easy to verify that, for a saturated state of yielding, the proposed microplane elasto-plastic model is equivalent to the classical von Mises or $J_{2}$ plasticity. Proof : From yield condition (49) we see that all the microplanes satisfy the condition $\sigma_{D}^{2}+\sigma_{T}^{2}=R^{2}$; this may then be introduced into the expression for $J_{2}$ in $(18 \mathrm{~b})$, which can then be integrated, furnishing

$$
2 J_{2}=\frac{3}{2 \pi} \int_{\Omega}\left(\sigma_{D}^{2}+\sigma_{T}^{2}\right) \mathrm{d} \Omega=\frac{3}{2 \pi} \int_{\Omega} R^{2} \mathrm{~d} \Omega=\frac{3}{2 \pi} 2 \pi R^{2}=3 R^{2} .
$$

This is the von Mises formulation for which the radius $r$ of the cylinder in the space of principal stresses is given by

$$
r=\sqrt{s_{1}^{2}+s_{2}^{2}+s_{3}^{2}}=\sqrt{2 J_{2}}=\sqrt{3} R .
$$

As indicated before, the yielding for proportional loading is reached first at some particular microplane, and then spreads gradually over the microplanes of all the other orientations. Macroscopically, this causes a sort of natural hardening as more and more microplanes join the yielding, until the $J_{2}$ plastic state is reached with a plateau in the macroscopic response. During the hardening regime, the response of the model is intrinsically anisotropic since some of the microplanes with given physical orientations are at yield while others are not. Therefore, the yield surface cannot be represented in the principal stress space during this phase.

It is possible, however, to find a principal stress representation for the condition of the first microplane yielding. To do that, one must first go back to the Mohr circle space. There, the stress states for all microplane orientations are represented by the classical Mohr circle, while the yield condition is represented by the circle (49). Starting from zero stresses, the yield circle of fixed radius $R$ will be centered at $\sigma_{N}=\sigma_{V}=0$, i.c., at the origin. With developing stresses, the Mohr circles will start growing and moving within the yield circle. The circle, at the same time, will be moving its center with $\sigma_{V}$. The first yielding will take place when the Mohr circle determined by $\sigma_{1}$ and $\sigma_{3}$ (the largest and smallest principal stresses) touches the yield circle. Simple geometrical considerations lead to the conclusion that the contact point must always be on the horizontal axis, either on the right side $\sigma_{N}=\sigma_{1}$ if $\sigma_{2}>\left(\sigma_{1}+\sigma_{3}\right) / 2$, or on the left side $\sigma_{N}=\sigma_{3}$ if $\sigma_{2}<\left(\sigma_{1}+\sigma_{3}\right) / 2$.

In the case with $\sigma_{2}=\left(\sigma_{1}+\sigma_{3}\right) / 2$, the largest Mohr circle and the yield circle share the same center. Therefore, the contact is simultaneous at all points of the circle. Restricting attention to the $1 / 48$ of the principal stress space in which $\sigma_{1} \geqslant \sigma_{2} \geqslant \sigma_{3} \geqslant 0$, the tangency conditions for the two cases depending on $\sigma_{2}$ can be written as follows :

$$
\begin{aligned}
& \text { if } \sigma_{2} \geqslant \frac{\sigma_{1}+\sigma_{3}}{2} \rightarrow \sigma_{1}=\sigma_{V}+R \\
& \text { if } \sigma_{2} \leqslant \frac{\sigma_{1}+\sigma_{3}}{2} \rightarrow \sigma_{3}=\sigma_{V}-R .
\end{aligned}
$$

In the principal stress space, these linear inequalities represent a prism parallel to the $p$ axis, with a regular hexagonal cross-section on the $\pi$-plane. This hexagon is fully within the von Mises circle of radius $r$, with inner radius $r / \sqrt{2} \doteq 0.707 r$, outer radius 


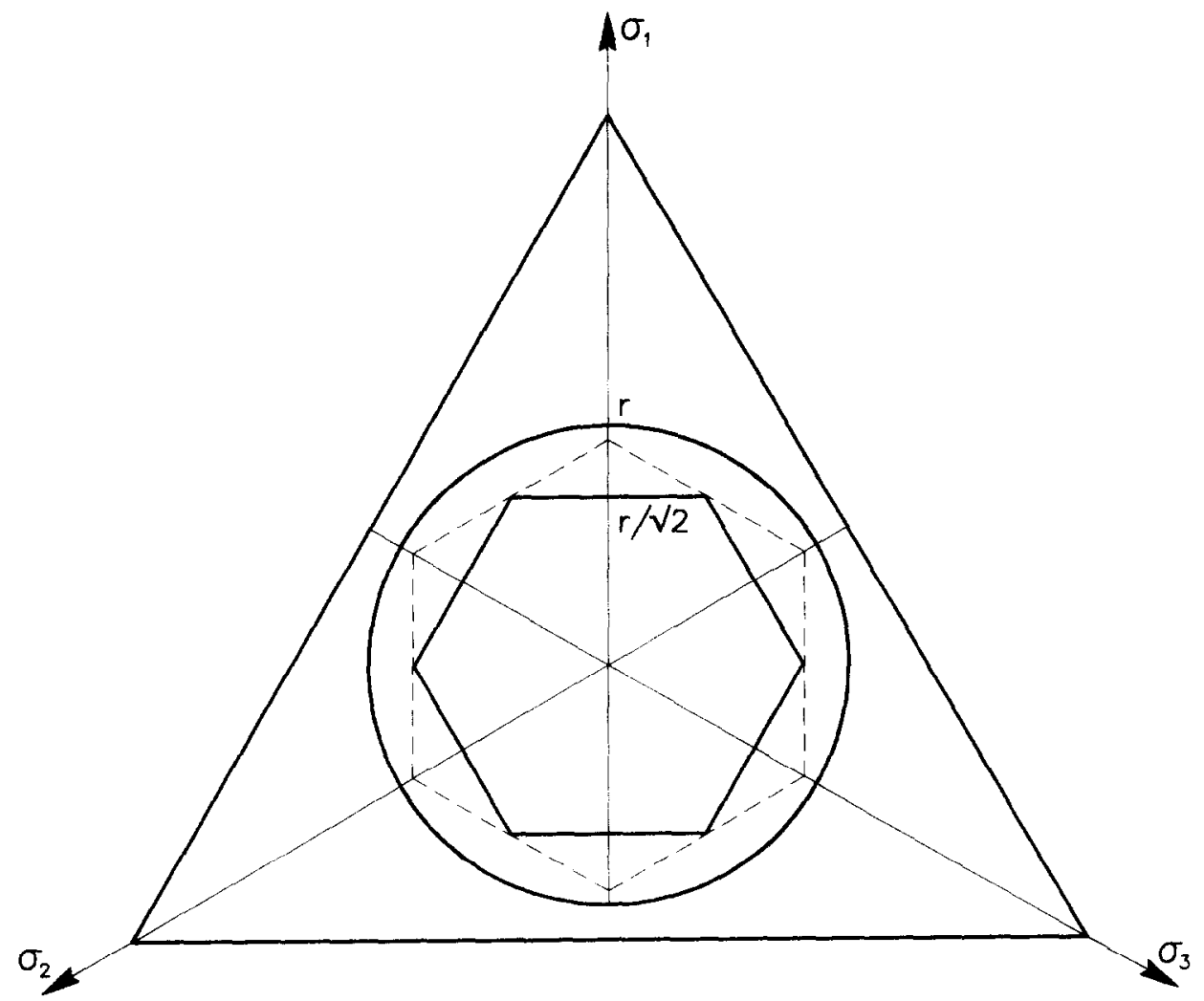

Fig. 2. Microplane elasto-plastic model: resulting surfaces for initial yielding (inner hexagon), saturated yielding (outer circle) and reference Tresca criterion (dashed hexagon) in the principal stress space.

$\sqrt{2 / 3} r=0.816 r$ and vertices at 30 on each side of the vertical $\sigma_{1}$-axis, as shown in Fig. 2 . While having the same shape, the hexagon is rotated $30^{\circ}$ with respect to the classical Tresca criterion, which has one vertex on the vertical axis of the $\pi$-plane itself. This is caused by one essential difference in the underlying yield condition for a generic plane. In the Tresca criterion, the yield condition is defined with a limit value $c$ for the radius of the largest Mohr circle itself. So, one can consider that the yield condition is represented by a circle of fixed radius $c$ which always has the same center as the largest Mohr circle, at $\left(\sigma_{1}+\sigma_{3}\right) / 2$. But in the present microplane model, the center of the yield circle is at $\sigma_{V}=\left(\sigma_{1}+\sigma_{2}+\sigma_{3}\right) / 3$, and so it is influenced by $\sigma_{2}$. Only when $\sigma_{2}=\left(\sigma_{1}+\sigma_{3}\right) / 2$, both circles have the same center, and the models become equivalent if $c=R$. In the $\pi$-plane representation (Fig. 2), this situation corresponds to the vertices of the microplane hexagon; these coincide with the midpoints of the sides of the corresponding Tresca criterion prism with $c=R$, which is shown in the figure by dashed lines. The outer radius of the microplane hexagon, is then the same as the inner radius of the corresponding Tresca hexagon, which itself has an outer radius equal to $2 \sqrt{2} / 3 r=0.943 r$.

5.3.2. Kinematic constraint and strain decomposition. Because of the microplane elastoplastic relations (40)-(42) with $E_{D}^{0}=E_{T}^{0}$ (double constraint for the elastic model), the microplane yield condition (49) can be rewritten in terms of strains :

$$
F=\left(\varepsilon_{D}^{e}\right)^{2}+\left(\varepsilon_{T}^{e}\right)^{2}-\bar{R}^{2}=\left(\varepsilon_{D}-\varepsilon_{D}^{p}\right)^{2}+\left(\varepsilon_{T}-\varepsilon_{T}^{p}\right)^{2}-\bar{R}^{2}=0 ; \quad \bar{R}=\frac{R}{E_{T}^{0}}
$$

This is another circle of fixed radius $\bar{R}$ in the microplane shear-deviatoric strain space 


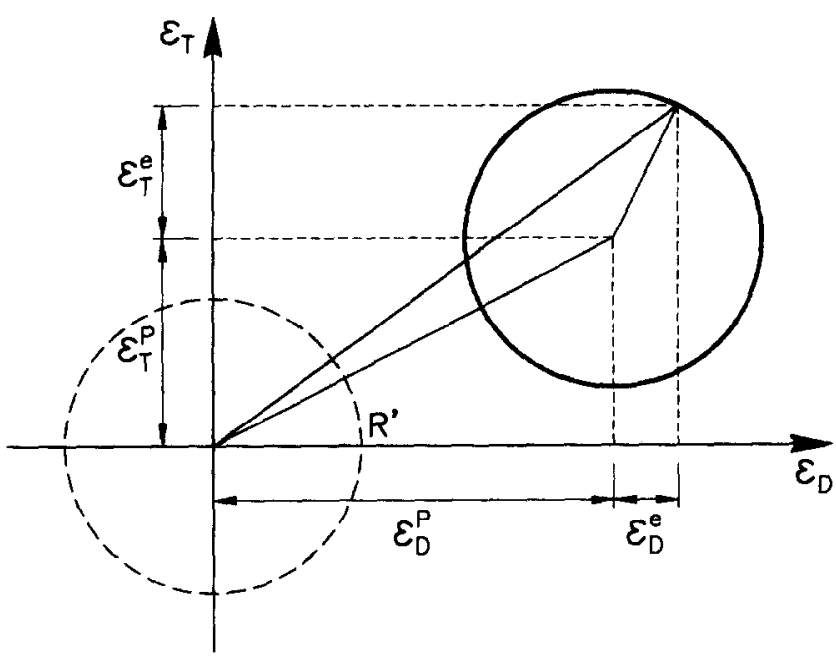

Fig. 3. Microplane elasto-plastic model: yield surface and elasto-plastic decomposition in the microplane strain space.

$\left(\varepsilon_{D}-\varepsilon_{T}\right)$. After plastic strains have developed, this circle is not centered at the origin anymore, but at point $\left(\varepsilon_{D}^{p}, \varepsilon_{T}^{p}\right)$ of that space (Fig. 3).

Now, the kinematic constraint is assumed. This means that, for a prescribed macroscopic strain history, the microplane strains $\varepsilon_{D}$ and $\varepsilon_{T_{r}}$ are always given. If the circular yield surface is to be satisfied, then as soon as the strain state reaches the current circle, the circle itself must be dragged along, in such a way that the current strain state point always lies on or inside the circle, but never outside. At each stage of the process, the position of the center of the circle represents the current plastic strains $\varepsilon_{D}^{p}$ and $\varepsilon_{T}^{p}$, and the vector from that point to the current strain state represents the elastic strains $\varepsilon_{D}^{e}$ and $\varepsilon_{T}^{e}$.

There are various possible ways to move the circle around, which in a loose analogy with various possible flow rules of the microplane plastic model can be considered. The following procedure may be adopted: the circle is moved in such a way that its center always remains on the straight line connecting the previous center and the new strain state outside the previous circle. In the case that the strain increment takes place along a direction radial from the current center (i.e., normal to the circle), the center itself will move along the same direction. In the case that infinitesimal increments are not normal to the circle, the trajectory of the center would be a curved line, resulting from straight infinitesimal segments.

5.3.3. Discussion and results for uniaxial tension-compression test. One important feature of the model in the way it has been defined is that, for prescribed strain increments, it is fully explicit, i.e., no numerical integration (such as commonly required in classical plasticity) is needed in this case. From the external strain increment, one can calculate the increments of microplane strains, and from them the new position of the circles on each microplane. Thus, one can obtain the elastic microplane strains, then the microplane stresses, and finally the macroscopic stresses by integration over the hemisphere. Another important advantage of the model is that it automatically accounts for intrinsic anisotropy with a non-fully plastic response for non-proportional loading paths. The importance of this was already pointed out by Batdorf and Budiansky (1949) in their early proposal of a model with a similar philosophy.

To illustrate the behavior of the model, the stress-strain curves resulting from one cycle of a uniaxial compression-tension test are plotted in Fig. 4. An integration rule over the hemisphere with 28 sample orientations in which microplane variables are stored and updated has been used. Natural hardening for progressive plastification of the microplanes is apparent in the results, as well as the final plateau that corresponds to von Mises plasticity when all the microplanes are yielding. Exactly the same results but shifted by the distance $\sigma_{V}$ are obtained if the volumetric stresses are applied prior to the uniaxial load. 


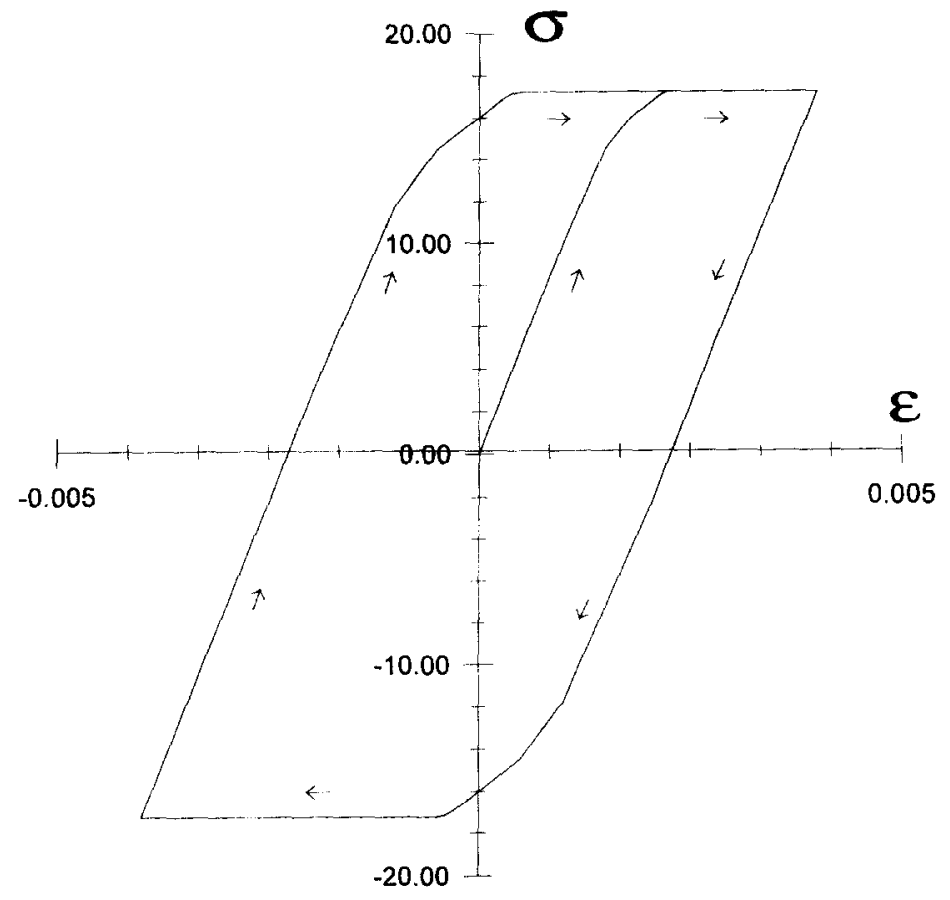

Fig. 4. Microplane elasto-plastic model : stress-strain curves obtained for uniaxial loading.

\section{FORMULATIONS WITH DAMAGE AND THE ENERGY EQUIVALENCE APPROACH}

\subsection{Continuum damage mechanics and the energy equivalence approach}

Damage can be conveniently introduced into the kinematically constrained microplane formulation according to the classical continuum damage mechanics (CDM) (Kachanov, 1958; Rabotnov, 1969; Lemaitre and Chaboche, 1985), based on the concepts of effective stresses and effective strains. On the continuum level, the effective stresses and strains are represented by tensors $\sigma_{i j}^{e f f}$ and $\varepsilon_{i j}^{e f f}$, which, in general, take values different from the apparent or nominal stress and strain $\sigma_{i j}$ and $\varepsilon_{i j}$. On the microplane level, effective stress $\sigma_{N}^{e f f}, \sigma_{V}^{e f f}, \sigma_{D}^{e f f}=\sigma_{N}^{e f f}-\sigma_{V}^{e f f}, \sigma_{T}^{e f f}$, and strains $\varepsilon_{N}^{e f f}, \varepsilon_{V}^{e f f}, \varepsilon_{D}^{e f f}=\varepsilon_{N}^{e f f}-\varepsilon_{V}^{e f f}$ and $\varepsilon_{T_{r}}^{e f f}$, are also considered, in addition to their nominal counterparts. The nominal and effective quantities at each level are then related with the help of damage variables. According to the classical CDM, this can be done in the context of one of the following three possible approaches: (i) the strain equivalence approach, (ii) the stress equivalence approach, and (iii) the energy equivalence approach.

In the first approach, effective strains are defined to be equal to their nominal counterparts, thus restricting the role of damage variables to the relations between the nominal and effective stress quantities. This approach can be idealized with a parallel arrangement of elements, all subjected to the same strain. The elements are breaking away of the system as the damage progresses, and thus are reducing the stress-carrying area fraction of the material. This approach was already pursued in the context of microplane model by Carol et al. (1991).

In the second approach, the dual stress equivalence approach, the nominal and effective stresses are equal, and the damage concept relates the nominal and effective strain measures. This approach may be idealized with a series arrangement, and is rarely used in practice.

The third approach, the energy equivalence, is the most complex of the three, but exhibits certain advantages such as generating symmetric secant stiffness matrices (which is not guaranteed with the strain or stress equivalence approaches). This is the approach adopted here.

With the addition of effective stresses and strains at the microplane and macroscopic levels, the number of variables and formulas in the microplane formulation is increased considerably. It is nevertheless possible to keep a relatively clear picture of the system with 


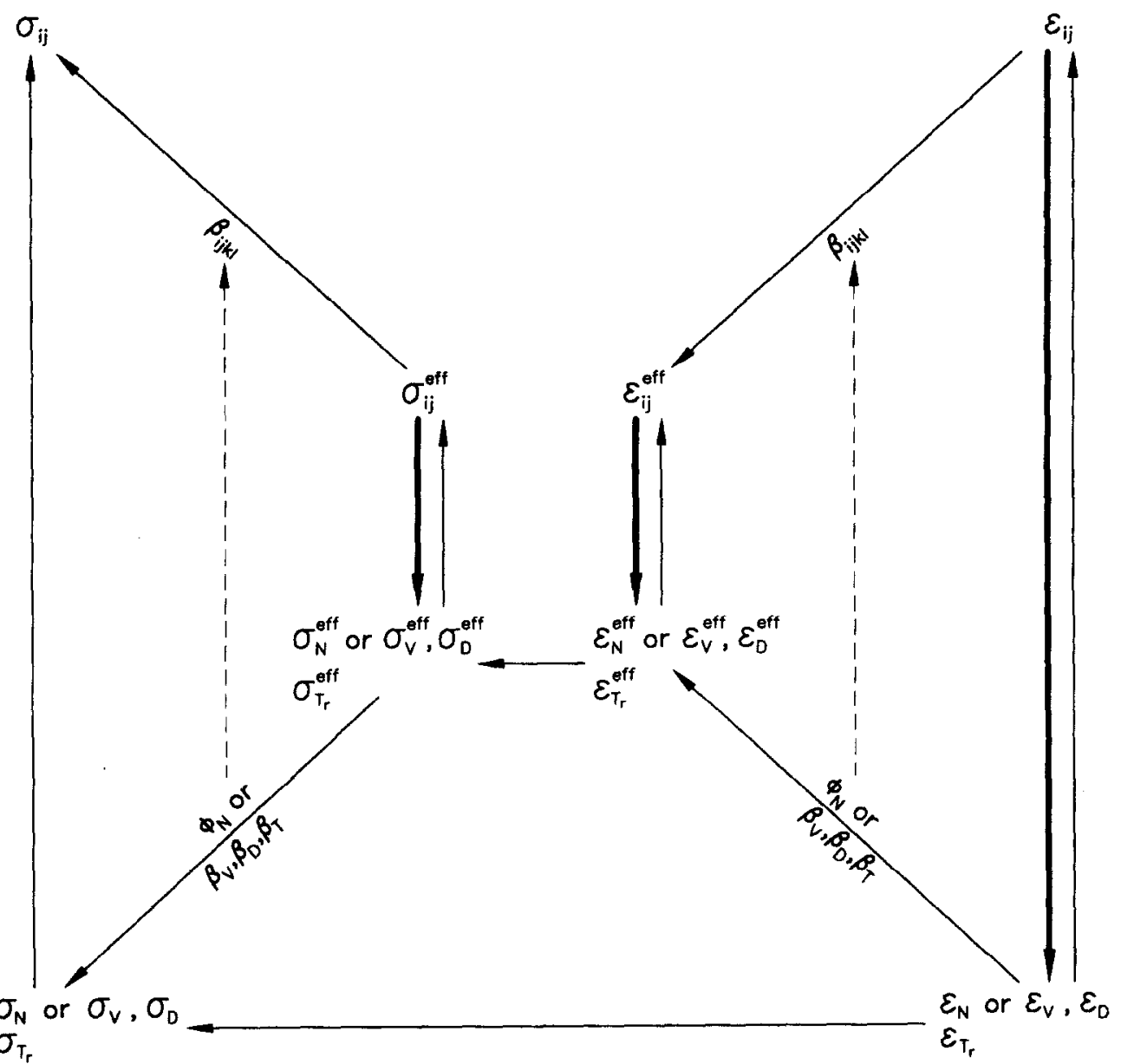

Fig. 5. Variables and relations in the microplane formulation with damage.

the help of the diagram in Fig. 5, which illustrates the properties and relations developed in this section. The outer rectangle in the figure represents the standard microplane model with kinematic constraint configured by the nominal (apparent) quantities. This part of the formulation preserves all the properties and relations described in the previous sections and Fig. 1. The four groups of effective quantities (stresses, strains, macro, micro) and their relations, configure a second microplane formulation represented by the inner rectangle in the figure.

The effective model can be assigned here the same meaning as in the classical CDM : that of representing the intrinsic behavior (rheology) of the material between microcracks. The effective quantities and their nominal counterparts are related through the damage variables. For energy equivalence, the relation between the nominal and effective strains is inverse to that between the nominal and effective stresses. Therefore, if the nominal and effective stress tensors, $\sigma_{i j}$ and $\sigma_{k l}^{e f f}$, are related linearly through a fourth-order damage tensor $\beta_{i j k l}$,

$$
\sigma_{i j}=\beta_{i j k l} \sigma_{k l}^{e f f} \quad \text { and } \quad \varepsilon_{i j}^{e f f}=\beta_{k l i j} \varepsilon_{k l}
$$

where $\varepsilon_{i j}$ and $\varepsilon_{i j}^{\text {eff }}$ are the corresponding nominal and effective strain tensors. Relations (54a) and $(54 \mathrm{~b})$ are presented by the inclined lines connecting the corresponding tensors in the upper part of Fig. 5.

The duality of the relations between stress and strain may be justified by enforcing the equivalence of elastic strain energy stored at the two levels (nominal and effective), although this requires some assumptions for the effective material model (e.g., linear elasticity). A 
more general derivation not requiring such assumptions can be given by considering how the external work is balanced between the overall model and its effective part. The external work rate to the overall model for a certain strain rate $\dot{\varepsilon}_{i j}$ is $\sigma_{i j} \dot{\varepsilon}_{i j}$. For fixed damage $\left(\beta_{i j k l}=\right.$ constant $)$, it is assumed that the external work rate is passed completely to the effective model, i.e., $\sigma_{i j} \dot{\varepsilon}_{i j}=\sigma_{i j}^{\text {eff }} \dot{\varepsilon}_{i j}^{\text {fff }}$. By substituting (54a) into this equation and eliminating $\sigma_{i j}^{\text {eff }}$, one obtains $\dot{\varepsilon}_{i j}^{\text {eff }}=\beta_{k i j} \dot{\varepsilon}_{k l}$, which is a relation strictly required only for increments with constant damage. This requirement can however be ensured by assuming the general relation (54b), which is in this sense a sufficient condition.

Because of its general character, however, eqn (54b) has additional implications, which relate to the energy dissipation associated with damage evolution. In the general case in which damage varies, the nominal-effective energy balance equation is defined as $\sigma_{i j} \dot{\varepsilon}_{i j}=\sigma_{i j}^{\text {eff }} \dot{\varepsilon}_{i j}^{\text {eff }}+\dot{D}^{d}$, where $\dot{D}^{d}$ is the damage energy dissipation rate which must be positive (i.e., the external work supply is not transferred entirely to the effective model, and the difference may define the damage dissipation). Equation (54b) can now be differentiated as $\dot{\varepsilon}_{i j}^{e f f}=\beta_{k l i j} \hat{\varepsilon}_{k l}+\dot{\beta}_{k l i j} \varepsilon_{k l}$, and this can be substituted into the balance equation, which leads to the fundamental expression for the damage dissipation rate in the models based on the energy equivalence approach :

$$
\dot{D}^{d}=-\varepsilon_{i j} \dot{\beta}_{i j k l} \sigma_{k j}^{\text {eff }} \geqslant 0
$$

The special case of this expression for linear elastic effective behavior can be obtained easily. The result agrees with alternative developments in the literature on elastic stiffness degradation and damage. For linear elasticity, $\sigma_{i j}^{e f f}=E_{i j k l}^{0} e_{k i}^{e f f}$. Substituting this and (54b) into (55) and defining the secant stiffness as shown in subsequent Section 6.2.5, eqn (69b), one obtains $\dot{D}^{d}=-\varepsilon_{i j} \dot{E}_{i j k l} \varepsilon_{k l} / 2$ which is the same as given in eqn (30a) of Carol et al. (1994b) for elastic-degrading materials.

Relations similar to $(54 \mathrm{a}, \mathrm{b})$, but without indices, hold if the stresses and strains are scalar quantities. This is the case for the individual microplane components and their damage coefficients. These relations correspond to the inclined lines connecting the nominal and effective microplane stresses and strains in the lower part of Fig. 5. There are, however, various ways to define the microplane damage coefficients and the corresponding nominaleffective relations. There are two main possibilities, which follow from the formulations with and without the split. We discuss them next.

\subsection{Model with fourth-order damage tensor and volumetric-deviatoric split}

6.2.1. Assumptions. One possibility to define the microplane damage variables and the corresponding nominal-effective relations is as follows:

$$
\sigma_{V}=\beta_{V} \sigma_{V}^{e f f} ; \quad \sigma_{D}=\beta_{D} \sigma_{D}^{e f f} ; \quad \sigma_{T_{r}}=\beta_{T} \sigma_{T_{r}}^{e f f}
$$

in which $\beta_{V}, \beta_{D}$ and $\beta_{T}$ are three microplane scalar damage coefficients varying from 1 to 0 . This is the same idea as developed earlier in the context of the strain equivalence (Carol et al., 1991), In the present context of energy equivalence, relations dual to the previous ones for stresses also apply for strains :

$$
\varepsilon_{V}^{\varepsilon f \prime}=\beta_{V} \varepsilon_{V} ; \quad \varepsilon_{D}^{e f f}=\beta_{D}^{\varepsilon_{D}} ; \quad \varepsilon_{T_{r}}^{u f f}=\beta_{T} \varepsilon_{T_{r}}
$$

This further requires that the effective microplane material laws must have the form:

$$
\sigma_{V}^{e f f}=\mathscr{F}_{V}^{\text {eff }}\left(\varepsilon_{V}^{\text {eff }}\right) ; \quad \sigma_{D}^{\text {eff }}=\mathscr{F}_{D}^{\text {eff }}\left(\varepsilon_{D}^{\text {eff }}\right) ; \quad \sigma_{T_{r}}^{\text {eff }}=\mathscr{F}_{T_{r}}^{\text {eff }}\left(\varepsilon_{T}^{\text {eff }}, \varepsilon_{V}^{e f f}, \varepsilon_{D}^{e f f}\right)
$$

It is now assumed that the efrective constitutive model exhibits the double constraint, i.e., that it satisfies the relations: 


$$
\begin{aligned}
& \varepsilon_{V}^{\text {eff }}=\frac{\varepsilon_{k k}^{e f f}}{3} ; \quad \varepsilon_{D}^{e f f}=\left(n_{i} n_{j}-\frac{\delta_{i j}}{3}\right) \varepsilon_{i j}^{e f f} ; \quad \varepsilon_{r_{r}}^{\text {eff }}=\frac{1}{2}\left(n_{i} \delta_{j r}+n_{j} \delta_{i r}-2 n_{i} n_{j} n_{r}\right) \varepsilon_{i j}^{\text {eff }} \quad(59 \mathrm{a}, \mathrm{b}, \mathrm{c}) \\
& \sigma_{V}^{e f f}=\frac{\sigma_{k k}^{e f f}}{3} ; \quad \sigma_{D}^{e f f}=\left(n_{i} n_{j}-\frac{\delta_{i i}}{3}\right) \sigma_{i j}^{e f f} ; \quad \sigma_{T_{r}}^{e f f}=\frac{1}{2}\left(n_{i} \delta_{j r}+n_{j} \delta_{i r}-2 n_{i} n_{j} n_{r}\right) \sigma_{i j}^{e f f} \quad(60 \mathrm{a}, \mathrm{b}, \mathrm{c})
\end{aligned}
$$

and also the corresponding integral equations:

$$
\begin{aligned}
& \sigma_{i j}^{e f f}=\sigma_{V}^{e f f} \delta_{i j}+\frac{3}{2 \pi} \int_{\Omega} \sigma_{D}^{e f f} n_{i} n_{j} \mathrm{~d} \Omega+\frac{3}{2 \pi} \int_{\Omega} \frac{\sigma_{T_{i}}^{e f f}}{2}\left(n_{i} \delta_{r j}+n_{j} \delta_{r i}\right) \mathrm{d} \Omega \\
& \varepsilon_{i j}^{e f f}=\varepsilon_{i j}^{e f f} \delta_{i j}+\frac{3}{2 \pi} \int_{\Omega} \varepsilon_{D}^{e f f} n_{i} n_{j} \mathrm{~d} \Omega+\frac{3}{2 \pi} \int_{\Omega} \frac{\varepsilon_{T_{r}}^{e f f}}{2}\left(n_{i} \delta_{r j}+n_{j} \delta_{r i}\right) \mathrm{d} \Omega .
\end{aligned}
$$

Relations (59) and (60) are represented in Fig. 5 by bold downward arrows connecting the effective stress and strain tensors to the effective microplane stresses and strains. Relations (61) and (62) are indicated by thin upward arrows parallel to the bold ones.

Developments in the following sections require only the integral expressions and the static constraint (60). But there is the general additional requirement that, for the particular situation of no damage, both the overall and effective models must coincide, i.e., the effective model must also satisfy the kinematic constraint. This requirement may also be extended to situations in which the damage is non-zero but all microcracks are closed due to load reversal. Therefore, it seems reasonable to restrict consideration to those effective models that satisfy the double constraint at all times. The elastic and elasto-plastic microplane formulations have been shown to do so, and therefore both are suitable candidates as effective models. Nevertheless, other formulations could be used as long as they satisfy the double constraint.

6.2.2. Relations between stresses and fourth-order damage tensor. Considering first only the relations between the four groups of stress quantities (macro, micro, nominal and effective stresses; see the left "box" in Fig. 5), one can start from the integral expression (9), substitute the microplane stresses in terms of the effective and damage counterparts (56), do the same with the macroscopic stresses (54a) substitute the effective static constraint $(60)$, and finally eliminate $\sigma_{k}^{e f f}$ from both sides of the expression. After some manipulations, one obtains

$$
\begin{aligned}
\beta_{i j k l}=\frac{\beta_{V}}{3} \delta_{i j} \delta_{k l}+\frac{3}{2 \pi} \int_{\Omega} \beta_{D} n_{i} n_{j}\left(n_{k} n_{l}-\frac{\delta_{k l}}{3}\right) \mathrm{d} \Omega \\
+\frac{3}{2 \pi} \int_{\Omega} \frac{\beta_{T}}{4}\left(n_{i} n_{k} \delta_{j l}+n_{i} n_{l} \delta_{i k}+n_{j} n_{k} \delta_{i l}+n_{j} n_{l} \delta_{i k}-4 n_{i} n_{j} n_{k} n_{l}\right) \mathrm{d} \Omega .
\end{aligned}
$$

So the fourth-order damage tensor is expressed as an integral of the scalar damage coefficients over all possible microplane orientations. Equation (63) is analogous to that obtained previously in the context of strain equivalence approach (Carol et al., 1991). Dual developments with the four types of strain quantities, involving integral expression (11), effective-to-nominal relations (54b) and (57), and overall kinematic constraint (8), lead to the same expression as (63) for the overall fourth-order tensor $\beta_{i j k l}$ that relates $\varepsilon_{i j}^{e f f}$ to $\varepsilon_{k l}$ (54b). This might have been expected in the energy equivalence approach. Relation (63) is represented by two dashed thin arrow lines connecting from bottom to top the corresponding quantities in Fig. 5. 
6.2.3. Relations between material laws. Microplane material laws that relate the nominal microplane stresses $\sigma_{V^{\prime}}, \sigma_{D}$ and $\sigma_{T}$ to the nominal microplane strains $\varepsilon_{V}, \varepsilon_{D}$ and $\varepsilon_{T r}$, can be deduced from the effective microplane laws (58) and the microplane damage relations (56) and (57). This is done by substituting (57) and (58) into (56), which yields the relations

$$
\sigma_{1}=\beta_{1} \mathscr{F}_{i f}^{e f}\left(\beta_{V} \varepsilon_{V}\right) \quad \sigma_{D}=\beta_{D} \cdot \mathscr{F}_{D}^{e f f}\left(\beta_{D} \varepsilon_{D}\right) \quad \sigma_{T_{r}}=\beta_{T} \mathscr{F}_{T_{r}}^{\text {eff }}\left(\beta_{T} \varepsilon_{T}, \beta_{V} \varepsilon_{V}, \beta_{D} \varepsilon_{D}\right) .
$$

$(64 a, b, c)$

Comparison of material laws (64) with their original microplane counterparts (10) immediately suggests that, in order to maintain full equivalence, the following relations should also exist :

$$
\beta_{1}=\mathscr{G}_{1}\left(\varepsilon_{1}\right) \quad \beta_{D}=\mathscr{G}_{D}\left(\varepsilon_{D}\right) \quad \beta_{T}=\mathscr{G}_{T}\left(\varepsilon_{T}, \varepsilon_{V}, \varepsilon_{D}\right)
$$

i.e., the microplane damage coefficients should be functions of their corresponding strains. This was exploited in a previous study (Carol et al., 1991), where the effective model was chosen as linear elasticity, and the damage evolution laws were simply deduced imposing full equivalence on the stress-strain material laws used in the standard microplane model.

6.2.4. General discussion of the damage formulation with split. According to the philosophy just outlined, introduction of damage would be nothing else than a reformulation of the standard kinematically constrained microplane model with volumetric-deviatoric split described in Section 2.2. The original variables and relations unfold into products of damage and effective variables, uncovering a richer internal structure with the effective model, etc., which offers deeper insight into the formulation.

Also, this reformulation offers new possibilities to combine damage with other types of effective behavior different from elasticity. This stems from the fact that, even though we derived the formulation assuming the effective model to be a microplane model, we can combine the resulting damage tensor (63) with any other effective constitutive model (e.g., linear elasticity, classical elasto-plasticity, viscoelasticity, etc.). In that case, the microplane formulation may be regarded exclusively as a way to obtain the damage tensor. The flow of calculations in Fig. 5 would be: from the strains $\varepsilon_{i j}$ to the microplane strains $\varepsilon_{V}, \varepsilon_{D}$ and $\varepsilon_{T}$ with the kinematic constraint (8), from those to the microplane damage coefficients $\beta_{V}$, $\beta_{D}$ and $\beta_{T}$ with the damage laws (65), and from those to the damage tensor $\beta_{i j k l}$ using (63) (vertical dashed line in the figure). The remaining calculations would be carried out according to the classical CDM with energy equivalence : the effective strains $\varepsilon_{i j}^{e f f}$ would be obtained with (54b) from $\varepsilon_{i j}$, the effective constitutive model itself would provide $\sigma_{i j}^{e f}$, and finally $\sigma_{i j}$ would be calculated using (54a). This procedure was applied successfully by Carol and Bažant (1991) in the context of the strain equivalence approach, to combine the microplane damage tensor with aging viscoelasticity of concrete in the form of an aging Maxwell chain.

On the other hand, this approach does not enhance the data fitting capabilities of Bažant and Prat's (1988) standard microplane model with by correcting the excessive volumetric expansions predicted for large tensile strains (see Section 2.3).

Another aspect that does not seem appealing is the lack of clear physical interpretation of the three microplane damage coefficients $\beta_{V}, \beta_{D}$ and $\beta_{T}$. Usually, damage variables are interpreted in geometrical terms as some stress-carrying area fraction. From this intuitive viewpoint, the simplest approach to microplane damage would correspond to having a single area fraction coefficient relating all the normal and shear components of the nominal and effective stresses on a given microplane. But this simple concept does not make sense for the damage model with volumetric-deviatoric split. The reason is that this case would correspond to $\beta_{T}=\beta_{D}=\beta_{V}$, but since $\beta_{V}$ is unique for all the microplanes, this situation actually corresponds to the same damage for all the microplanes, i.e., some form of isotropic degradation. This is reflected in the expressions that can be obtained by progressive simplifications of the integral expression for $\beta_{i j k l}$ in eqn (63). By setting $\beta_{D}=\beta_{T}$ (different for each microplane), one obtains 


$$
\beta_{i j k l}=\frac{\beta_{V}}{3} \delta_{i j} \delta_{k l}+\frac{3}{2 \pi} \int_{\Omega} \frac{\beta_{T}}{4}\left(-4 n_{i} n_{j} \frac{\delta_{k l}}{3}+n_{i} n_{k} \delta_{j l}+n_{i} n_{l} \delta_{j k}+n_{j} n_{k} \delta_{i l}+n_{j} n_{l} \delta_{i k}\right) \mathrm{d} \Omega
$$

which is the simplest form of the tensor still representing anisotropic damage. Further simplification falls already in the range of isotropic damage, for which $\beta_{D}=\beta_{T}=\beta_{V}=$ constant for all the microplanes, and

$$
\beta_{i j k l}=\frac{\beta_{V}}{2}\left(\delta_{i k} \delta_{j l}+\delta_{i l} \delta_{j k}\right)
$$

The aforementioned disadvantages of the microplane damage formulation with volumetric-deviatoric split and fourth-order damage tensor disappear in the alternative formulation without split and with a second-order tensor described in Section 6.3.

6.2.5. Particular formulation with linear elastic effective model. The simplest effective model is the linear elasticity which, according to the previous discussion, may be introduced directly with the classical relations

$$
\sigma_{i j}^{e f f}=E_{i j k l}^{0} \varepsilon_{k l}^{e f f} ; \quad E_{i j k l}^{0}=\lambda^{0} \delta_{i j} \delta_{k l}+\mu^{0}\left(\delta_{i k} \delta_{j l}+\delta_{i l} \delta_{j k}\right)
$$

Substituting (54b) into (68a) and the result into (54a), one obtains

$$
\sigma_{i j}=E_{i j k l} \varepsilon_{k l} ; \quad E_{i j k l}=\beta_{i j p q} E_{p q r s}^{0} \beta_{k l r s}
$$

which can also be expressed as :

$$
E_{i j k l}=\Omega_{i j k l p q r s} E_{p q r s}^{0} ; \quad \Omega_{i j k l p q r s}=\beta_{i j p q} \beta_{k l r s}
$$

This is the secant stiffness tensor of an elastic-damage formulation in which unloading always leads back to the origin of the $\sigma-\varepsilon$ space. Note that, due to the doubly contracted product with the damage tensor, the resulting secant stiffness is always symmetric (major symmetry), even if $\beta_{i j k l}$ itself is not (pairs of indices $i j$ and $k l$ are not interchangeable in eqns 63). This is a general result for damage formulations based on the energy approach, in contrast to those based on the strain equivalence or stress equivalence approaches. Symmetry of the secant stiffness is actually a fundamental requirement which has often been disregarded in the literature. Formulations with constant unloading-reloading stiffness should exhibit a wcll-defined cnergy potential in order to avoid spurious dissipation or generation of energy upon closed-loop load histories (Carol et al., 1994b). In models that take into account the recovery of stiffness due to microcrack closure, the arguments become more complicated, but a condition of this type should be a basic requirement for the underlying damage model (Carol and Willam, 1996).

Although not strictly necessary for the formulation, additional insight may be gained by considering linear elasticity as the result of an elastic microplane formulation with normal-deviatoric split and double constraint. This means rewriting the constitutive relations (64) in the form $\mathscr{F}_{V}^{e f f}\left(\varepsilon_{V}^{e f f}\right)=E_{0}^{V} \varepsilon_{V}^{e f f}$, etc., which yields

$$
\begin{aligned}
& \sigma_{V}=E_{V} \varepsilon_{V} ; \quad E_{V}=\beta_{V}^{2} E_{V}^{0} \\
& \sigma_{D}=E_{D} \varepsilon_{D} ; \quad E_{D}=\beta_{D}^{2} E_{D}^{0} \\
& \sigma_{T_{r}}=E_{T} \varepsilon_{T_{r}} ; \quad E_{T}=\beta_{T}^{2} E_{T}^{0}
\end{aligned}
$$

As already explained in Section 6.2.3, this formulation can be considered as a reformulation of the standard microplane model described in Section 2.2, for which the material laws (10) 
take the form of eqns (71)-(73). In eqns (71)-(73), $E_{V}, E_{D}$ and $E_{T}$ are the current secant microplane stiffnesses (assumed constant upon unloading and reloading), and the initial moduli $E_{\nu}^{0}, E_{D}^{0}$ and $E_{I}^{0}$ are given by (39).

Equations (71)-(73) are similar to eqns (15a, b, c) in Carol et al. (1991), in which analogous developments were presented within the framework of the strain equivalence approach instead of the energy equivalence approach. The damage variables in the two approaches are related as $\beta_{V}^{2}=\alpha_{V}, \beta_{D}^{2}=\alpha_{D}$ and $\beta_{T}^{2}=\alpha_{T}$. It follows that the corresponding fourth-order damage tensors are related as $\beta_{i j p q} \beta_{k i r s}=\alpha_{i j p q} \delta_{k r} \delta_{l s}$.

6.2.6. Particular formulation with elasto-plastic effective model. An elasto-plastic effective model is characterized by the classical equations

$$
\sigma_{i j}^{e f f}=E_{i j k l}^{0}\left(\varepsilon_{k l}^{e f f}-\varepsilon_{k l}^{e f f, p}\right)
$$

Substituting (54b) into this equation and the result into (54a), one obtains

$$
\sigma_{i j}=E_{i j k l}\left(\varepsilon_{k l}-\varepsilon_{k l}^{p}\right)
$$

where $E_{i j k l}$ is the same secant stiffness as in $(69 \mathrm{~b})$, and the nominal plastic strains are given by

$$
\varepsilon_{k l}^{p}=\left[\boldsymbol{\beta}^{-1}\right]_{k l p q} \varepsilon_{p q}^{e f f, p}
$$

$\left[\boldsymbol{\beta}^{-1}\right]_{k i p q}$ denotes a fourth-order tensorial grouping of the coefficient of the $6 \times 6$ matrix $\boldsymbol{\beta}^{-1}$, $\beta$ being the $6 \times 6$ matrix formed in the standard way from $\beta_{i j k l}$.

Equations (74)-(76) with the fourth-order damage tensor $\beta_{i j k l}$ given by (63) suffice to combine the microplane damage formulation with the classical elasto-plasticity as the cffective model. One can, however, additionally consider that the elasto-plastic effective model consists of a microplane elasto-plastic formulation with the split and with a double constraint, of the general type described in Section 5. This means that one can write the following microplane effective constitutive laws

$$
\sigma_{V}^{e f f}=E_{V}^{0}\left(\varepsilon_{V}^{e f f}-\varepsilon_{V}^{e f f, p}\right) ; \quad \sigma_{D}^{e f f}=E_{D}^{0}\left(\varepsilon_{D}^{e f f}-\varepsilon_{D}^{e f f, p}\right) ; \quad \sigma_{T_{r}}^{e f f}=E_{T}^{0}\left(\varepsilon_{T_{r}}^{e f f}-\varepsilon_{T_{r}}^{e f, p}\right) \quad(77 \mathrm{a}, \mathrm{b}, \mathrm{c})
$$

and the micro-macro relation

$$
\varepsilon_{i j}^{e f f, p}=\varepsilon_{V}^{e f f, p} \delta_{i j}+\frac{3}{2 \pi} \int_{\Omega} \varepsilon_{D}^{e f f, p} n_{i} n_{j} \mathrm{~d} \Omega+\frac{3}{2 \pi} \int_{\Omega} \frac{\varepsilon_{T_{r}}^{e f f, p}}{2}\left(n_{i} \delta_{r j}+n_{j} \delta_{r i}\right) \mathrm{d} \Omega
$$

Equations (57) can now be substituted into (77) and the result in (56), obtaining

$$
\begin{array}{cc}
\sigma_{V}=E_{V}\left(\varepsilon_{V}-\varepsilon_{V}^{p}\right) ; & \varepsilon_{V}^{p}=\frac{1}{\beta_{V}} \varepsilon_{V}^{e f f . p} \\
\sigma_{D}=E_{D}\left(\varepsilon_{D}-\varepsilon_{D}^{p}\right) ; & \varepsilon_{D}^{p}=\frac{1}{\beta_{D}} \varepsilon_{D}^{e f f, p} \\
\sigma_{T_{r}}=E_{T}\left(\varepsilon_{T_{r}}-\varepsilon_{T_{r}}^{p}\right) ; & \varepsilon_{T_{r}}^{p}=\frac{1}{\beta_{T}} \varepsilon_{T_{r}}^{\text {eff.p. }} .
\end{array}
$$

Similarly as in the previous section, this formulation may be considered as a reformulation of the standard microplane model (Section 2.2), in which the microplane laws (10) take the form of (79)-(81). With microplane elasto-plasticity as the effective model, the 
general macroscopic expression (75) holds. In that equation, the secant stiffness is given by (69b), and the macroscopic plastic strain is given by the integral eqn (47) where $\varepsilon_{V}^{p}, \varepsilon_{D}^{p}$ and $\varepsilon_{T,}^{p}$ are given by $(79 b)-(81 b)$.

If the elasto-plastic microplane effective model exhibits the double constraint, the plastic effective strains satisfy additional relations. A kinematic micro-macro constraint applies between the effective plastic strains (see Section 5.2);

$$
\begin{aligned}
& \varepsilon_{V}^{e f f, p}=\frac{\delta_{i j}}{3} \varepsilon_{i j}^{e f, p} \\
& \varepsilon_{D}^{e f f, p}=\left(n_{i} n_{j}-\frac{\delta_{i j}}{3}\right) \varepsilon_{i j}^{e f, p} \\
& \varepsilon_{T}^{e f f, p}=\frac{1}{2}\left(n_{i} \delta_{r j}+n_{j} \delta_{r i}-2 n_{i} n_{j} n_{r}\right) \varepsilon_{i j}^{e f f, p} .
\end{aligned}
$$

Equations (79b)-(81b) may then be substituted into the integral eqn (47) with $\varepsilon_{b}^{e f f, p}, \varepsilon_{D}^{e f f, p}$ and $\varepsilon_{f, p}^{e f, p}$ given before; $\varepsilon_{i j}^{p}$ on the left-hand side may then be replaced by (76) and $\varepsilon_{i j}^{e f f, p}$ eliminated from both sides. This yields

$$
\begin{aligned}
{\left[\boldsymbol{\beta}^{-1}\right]_{i j k l}=\frac{1}{3 \beta_{V}} \delta_{i j} \delta_{k l}+\frac{3}{2 \pi} \int_{\Omega} } & \frac{1}{\beta_{D}} n_{i} n_{j}\left(n_{k} n_{l}-\frac{\delta_{k l}}{3}\right) \mathrm{d} \Omega \\
& +\frac{3}{2 \pi} \int_{\Omega} \frac{1}{4 \beta_{T}}\left(n_{i} n_{k} \delta_{j l}+n_{i} n_{l} \delta_{j k}+n_{j} n_{k} \delta_{i l}+n_{j} n_{l} \delta_{i k}-4 n_{i} n_{j} n_{k} n_{l}\right) \mathrm{d} \Omega
\end{aligned}
$$

which is an integral relation analogous to (63), but for the inverse tensor $\beta^{-1}$ in terms of the inverses $1 / \beta_{V}, 1 / \beta_{D}$ and $1 / \beta_{T}$.

\subsection{Simpler model with second-order damage tensor without volumetric-deviatoric split}

This formulation provides a simpler and more easily understandable representation of damage, with a single damage variable for each microplane and a second-order damage tensor. The fact that the formulation is without volumetric-deviatoric split does not preclude combination with the effective microplane models with split, discussed in the preceding section. Rather, the combination of a damage model without split and an effective constitutive formulation with split can provide a smooth continuous transition between the two approaches, and this could be a natural alternative to the introduction of stress-strain boundaries which overcome the excessive volumetric strain caused by large uniaxial tension found in the original microplane model with split (see discussion in Section 2.3).

6.3.1. Assumptions In this alternative approach, a single scalar damage variable $\phi_{N}$ varying from 1 to 0 , is assumed for each microplane. The normal-effective stress relations read

$$
\sigma_{N}=\phi_{N} \sigma_{N}^{\text {eff }} ; \quad \sigma_{T_{r}}=\phi_{N} \sigma_{T_{r}}^{\text {eff }}
$$

These assumptions are essentially different from their counterparts with split. Actually, (84a) may be rewritten in terms of the volumetric and deviatoric components as $\sigma_{V}+\sigma_{D}=\phi_{N}\left(\sigma_{V}^{e f f}+\sigma_{D}^{\text {eff }}\right)$, which cannot be obtained as any particular combination of (56a) and (56b). In the context of energy equivalence, relations dual to (84) hold between the effective and nominal microplane strains

$$
\varepsilon_{N}^{e f f}=\phi_{N} \varepsilon_{N} ; \quad \varepsilon_{T_{r}}^{e f f}=\phi_{N} \varepsilon_{T_{r}}
$$

Some assumptions must now be made for the effective constitutive model. In the context of the damage formulation without volumetric-deviatoric split, it might seem more 
natural to assume an effective constitutive model also formulated without the split. But it was explained before that the effective constitutive model was required to exhibit a double constraint, and that this constraint could be achieved consistently only with the model with split. Therefore, we may base the derivation on the effective constitutive model with split, eqns (58)-(62) in Section 6.2.1. It must be noted, however, that the resulting damage tensor is the same independently of the choice of the microplane effective formulation and, similar to Section 6.2, once the damage tensor is developed, it can be combined with any other type of effective constitutive model (i.e., elasticity, classical elasto-plasticity, etc.).

6.3.2. Relations hetween stresses and second-order damage tensor. Considering first only relations between the four groups of stress quantities (macro, micro, nominal, effective), one can start from the integral expression (2) without split (which is also satisfied by the model with split), express the microplane stresses in terms of the damage variables and their effective counterparts (84), introduce the static constraint for effective stresses (60) taking into account the relation $\sigma_{N}=\sigma_{\nu}+\sigma_{D}$, substitute the fourth-order damage tensor-based relation (54a) between the stress and effective stress tensors, and finally eliminate $\sigma_{k l}^{\text {eff }}$ from both sides of the equation. After appropriate manipulations, one obtains the following expression for the fourth-order damage tensor

$$
\beta_{i, k l}=\frac{3}{2 \pi} \int_{\Omega} \frac{\phi_{N}}{4}\left(n_{i} n_{k} \dot{\delta}_{j l}+n_{i} n_{l} \dot{\delta}_{j k}+n_{j} n_{k} \delta_{i l}+n_{j} n_{l} \delta_{i k}\right) \mathrm{d} \Omega
$$

i.e., the fourth-order damage tensor is given as an integral of the scalar damage coefficient over all the possible microplane orientations. Note that this equation is obtained independently of whether the effective model is assumed with or without split. Note also that if $\phi_{N}$ is the same for all microplanes, one can bring it in front of the integral, evaluate the integral with the help of (5) and obtain $\beta_{i, k l}=\phi_{N}\left(\delta_{i k} \delta_{j l}+\delta_{i l} \delta_{j k}\right) / 2$ which is the same isotropic tensor as obtained in the damage model with split and with $\beta_{V}=\beta_{D}=\beta_{T}$ (67).

Now, a key step. Assume that the fourth-order tensor $\beta_{i j k l}$ is not the simplest tensorial measure of the damage state, but there exists a symmetric second-order tensor $w_{i j}$ such that

$$
\beta_{i j k l}=w_{i k} w_{j l}
$$

This may be substituted into the left-hand side of the preceding integral equation, and both sides multiplied by $\delta_{k l}$. After rearrangements, this yields the fundamental expression

$$
\phi_{i j}=w_{i k} w_{k j}=\frac{3}{2 \pi} \int_{\Omega} \phi_{N} n_{i} n_{j} \mathrm{~d} \Omega
$$

$\phi_{i j}$ may be called the second-order damage tensor, and $w_{i j}$ is the tensorial square root of $\phi_{i j}$, sharing the same principal directions and having square root principal values. In our notation, this is expressed as $w_{i j}=\left[\boldsymbol{\phi}^{1 / 2}\right]_{i j}$ or $\phi_{i j}=\left[\mathbf{w}^{2}\right]_{i j}$, which must not be confused with $\phi_{i j}^{1 / 2}$ or $w_{i j}^{2}$ (square roots or second power of specific components).

It must be noted that if the foregoing procedure is applied to $\beta_{i j k l}$ from the model with split (63), all the terms with $\beta_{D}$ and $\beta_{T}$ cancel out. The result is $\phi_{i j}=\beta_{b} \delta_{i j}$, i.e., isotropic degradation exclusively. From this, obviously no anisotropic fourth-order damage tensor can be formed.

Dual developments with the four types of strain quantities, involving integral expression (4), effective-to-nominal stress or strain relations (54b) and (85), and overall kinematic constraint (8), lead to the same fourth-order (86) and second-order damage tensors (88) as obtained for stresses.

6.3.3. Relations between material laws. For an effective microplane model without split, the relations between material laws in the effective and overall models would be analogous to (64) with only the two components $N$ and $T_{r}$. These relations could then be 
considered directly equivalent to the constitutive laws without the split (3), and the entire damage formulation with the second-order tensor could be considered as a reformulation of the original microplane model without split from Section 2.1. However, as explained before, in order to represent the entire range of Poisson ratios in the linear range and capture the volumetric-deviatoric coupling in the compressive nonlinear behavior, the effective constitutive model must have the volumetric-deviatoric split. In that case, the relations between the microplane strains and stresses take the form:

$$
\begin{aligned}
& \sigma_{N}=\phi_{N}\left[\mathscr{F}_{V}^{e f f}\left(\varepsilon_{V}^{\text {eff }}\right)+\mathscr{F}_{D}^{\text {eff }}\left(\varepsilon_{D}^{\text {eff }}\right)\right] \\
& \sigma_{T_{r}}=\phi_{N} \mathscr{F}_{T_{r}}^{\text {eff }}\left(\varepsilon_{T}^{\text {eff }}, \varepsilon_{V}^{\text {eff }}, \varepsilon_{D}^{\text {eff }}\right)
\end{aligned}
$$

where $\varepsilon_{V}^{e f f}$ and $\varepsilon_{D}^{e f f}$ cannot be replaced as in (64) with the products of their corresponding damage coefficients and nominal counterparts. Rather, one starts in this case by assuming an evolution law for the microplane damage coefficient $\phi_{N}$ in the form:

$$
\phi_{N}=\mathscr{G}_{N}\left(\varepsilon_{N}, \boldsymbol{\varepsilon}_{T}\right)
$$

The flow of calculations, in the context of microplane effective model, is then as follows : First, for each microplane the values of $\varepsilon_{N}$, and $\varepsilon_{T_{r}}$ are obtained from $\varepsilon_{i j}$ using (1), and $\phi_{N}$ using (90); second, $\phi_{i j}$ is calculated from (88), its square root $w_{i j}$ and $\beta_{i j k l}$ from (87); then, the effective strain tensor $\varepsilon_{i j}^{e f f}$ is oblained from $\varepsilon_{i j}$ using $(54 \mathrm{~b})$ and, finally, $\varepsilon_{V}^{e f f}, \varepsilon_{D}^{e f f}$ and $\varepsilon_{T_{r}}^{e f f}$ are obtained from the kinematic constraint equations for the effective model (59) (note in this case the strict requirement for the double constraint at all times in the effective constitutive model; also note that $\varepsilon_{T_{r}}^{e f f}$ is not affected by the split, and thus the preceding procedure will give the same result as the direct product $\phi_{N} \varepsilon_{T_{r}}$ ). After that, (89) and (2) are used to obtain $\sigma_{i j}$.

The preceding procedure may be abbreviated if the effective constitutive model is not a microplane formulation, but some classical constitutive model such as elasticity or elastoplasticity. In that case, the same procedure is followed until $\varepsilon_{i j}^{e f f}$ is obtained. Then, $\sigma_{i j}^{e f f}$ is calculated from the effective constitutive model, and finally $\sigma_{i j}$ is obtained using (54a).

The overall formulation combining the damage model and the effective constitutive model in the way described is not equivalent to the standard microplane model with or without split. It actually represents a certain combination of both, such that the behavior will be closer to the model with split when the damage is not activated and the effective model dominates the overall behavior, and closer to the model without split in the opposite case.

6.3.4. Further considerations on damage formulation without split and second-order damage tensor. As a second-order tensor, $\phi_{i j}$ is perhaps the simplest consistent form of representing anisotropic damage (vectors have been proposed, but they do not satisfy basic requirements such as being able to represent isotropic damage and other more theoretical requirements, see Leckie and Onat, 1981). For the case of no damage $\left(\phi_{N}=1\right.$ for all microplanes), the integral (5) can be evaluated in a closed form, yielding $\phi_{i j}=\delta_{i j}$. For full damage ( $\phi_{N}=0$ for all microplanes), $\phi_{i j}$ becomes the null second-order tensor. For intermediate situations, $\phi_{i j}$ is a tensor with principal values between 1 and 0 . The principal directions of damage can be easily obtained, and can be interpreted as the physical directions of maximum and minimum degradation. A graphical representation of $\phi_{i j}$, similar to that commonly used for representing stresses or strains, is possible.

Due to all these convenient properties, the microplane theory with the second-order damage tensor provides a relatively simple and convenient framework for material degradation. In fact, the final expression (88) is similar to some previous expressions for secondorder fabric tensors developed by applying various micromechanical and statistical arguments to microcrack systems in elastic materials (Kachanov, 1980; Oda, 1983). From a different perspective, the tensorial nominal-effective stress relations adopted, (54a) and (87), can be combined and rewritten in the form: 


$$
\sigma_{i j}=\left[\phi^{1 / 2}\right]_{i k} \sigma_{k l}^{e f f}\left[\phi^{1 / 2}\right]_{l i}
$$

where, as indicated before, $\left[\boldsymbol{\phi}^{1 / 2}\right]_{i j}$ denotes the $i j$ component of the square root matrix $\boldsymbol{\phi}^{1 / 2}$, $\phi$ being the $3 \times 3$ matrix of coefficients $\phi_{i j}$. Expression (91) corresponds to the pioneering proposal by Cordebois and Sidoroff (1982) for this kind of relation with second-order damage tensors.

The formulation proposed for $\phi_{i j}$ in (88) is the simplest possible within the microplane philosophy, with a single scalar damage parameter $\phi_{N}$ per microplane. More elaborate alternative formulations with second-order damage tensor can also be easily envisaged. One interesting possibility is to complement the "normal" microplane damage $\phi_{N}$ with a "tangential" term of vectorial nature $\phi_{T_{r}}$ (and therefore of a completely different nature to $\beta_{T}$ in previous sections). An inspiration can be found in the analogy of the microplane damage variables with the projections of $\phi_{i j}$ on the microplane (similar to the projections of the stress or strain tensor on a plane), having normal components $\phi_{i j} n_{i} n_{j}$ and tangential components $\phi_{i j} n_{j}-\left(\phi_{k} n_{k} n_{l}\right) n_{i}$. The tangential components $\phi_{T}$, must lie in the plane (i.e., $\left.\phi_{T_{r}} n_{r}=0\right)$ and represent the distortion that accompanies the stress-carrying area reduction given by $\phi_{N}$. With the new damage term, the nominal-effective relations read:

$$
\begin{gathered}
\sigma_{N}=\phi_{N} \sigma_{N}^{e f f}+\phi_{T_{r}} \sigma_{T_{r}}^{e f f} \\
\sigma_{T_{r}}=\phi_{T_{r}} \sigma_{N}^{e f f}+\phi_{N} \sigma_{T_{r}}^{e f f}
\end{gathered}
$$

i.e., a cross-effect is introduced between the normal and tangential components, such that a shear nominal stress can exist on a microplane on which there are only normal effective stresses, or vice versa. If the derivation of $\phi_{i j}$ is now repeated with this new relation instead of (84), one obtains the new integral expression

$$
\phi_{i j}=\frac{3}{2 \pi} \int_{\Omega} \phi_{N} n_{i} n_{j} \mathrm{~d} \Omega+\frac{3}{2 \pi} \int_{\Omega} \frac{\phi_{T_{r}}}{2}\left(n_{i} \delta_{r j}+n_{j} \delta_{r i}\right) \mathrm{d} \Omega
$$

which is analogous to the integral equation for stresses without split (2). This analogy can be useful to understand the changes induced in $\phi_{i j}$ by the addition of the tangential microplane damage term.

6.3.5. Particular formulation with linear elastic effective model. For a linear elastic effective model, we have again the same equations as (68)-(70) for the damage formulation with split. However, $\beta_{i j k}$ can now be further replaced with $w_{i k} w_{j l}(87)$, to yield:

$$
E_{i j k l}=w_{i p} w_{j q} w_{k r} w_{l s} E_{p q r s}^{0}
$$

This can also be expressed as

$$
E_{i j k l}=\Omega_{i j k l p q r s} E_{p q r s}^{0} ; \quad \Omega_{i j k l p q r s}=\beta_{i j p q} \beta_{k i r s}=w_{i p} w_{j q} w_{k r} w_{l s}
$$

Additionally, one can replace the expression for the initial stiffness (68b), develop all products and set $w_{i k} w_{k j}=\phi_{i j}$. This gives

$$
E_{i j l k}=\lambda^{0} \phi_{i j} \phi_{k l}+\mu^{0}\left(\phi_{i k} \phi_{j l}+\phi_{i l} \phi_{j k}\right)
$$

This macroscopic expression is equivalent to the elastic-damage model based on a secondorder damage tensor proposed by Valanis (1990), who called $\phi_{i j}$ the integrity tensor. The formulation is also related to the theories of other authors based on second-order damage tensors (Chow and Wang, 1987; Shen et al., 1989). 
Note also that (96) has the same form as the elastic stiffness tensor (68b), in which Kronecker deltas have been replaced with the integrity tensor $\phi_{i j}$. When there is no damage, $\phi_{i j}=\delta_{i j}$ and the initial elastic expression (68b) is recovered.

Same as for the model with split, the linear elastic effective model could now be considered equivalent to an elastic microplane model with volumetric-deviatoric split and a double constraint. In this case, however, substitution of relations similar to (33) and (39) into (84) does not provide direct equivalence with the material laws of any standard microplane formulation with or without split (it is actually a combination of both, see the discussion in Section 6.3.3). Little additional insight is gained in this way.

6.3.6. Particular formulation with elasto-plastic effective model. An elasto-plastic effective model may be characterized macroscopically by eqn (74), and its combination with damage by eqns (75)-(76), same as in the formulation with the fourth-order damage tensor. In this case, however, the fourth-order tensor $\beta_{i j k l}$ may be replaced by $w_{i k} w_{j l}(87)$, leading to eqns (94)-(96) for the secant stiffness $E_{i j k l}$, and to the following equation for the plastic strain:

$$
\varepsilon_{k l}^{p}=\left[\mathbf{w}^{-1}\right]_{k p} \varepsilon_{p q}^{e f f p}\left[\mathbf{w}^{-1}\right]_{q l}
$$

where $\left[\mathbf{w}^{-1}\right]_{k p}$ denotes the $k p$ component of $\mathbf{w}^{-1}$, inverse to the $3 \times 3$ matrix $\mathbf{w}$ of components $w_{i j}$.

\section{SUMMARY}

In the existing literature, the microplane model has been developed in various versions with different assumptions and modeling capabilities. Overall, the model has been shown capable of reproducing the salient features of quasibrittle materials such as concrete, rock and clay, under a multitude of different loading conditions. However, the fact that various versions of the model were presented, together with the lack of clear relations to more traditional damage and plasticity-based constitutive models, suggested the need for a more general theoretical framework in which all these relations could be clarified.

The classical assumptions of kinematic constraint with or without volumetric-deviatoric split of normal components have been reviewed, and some new arguments have been presented on how to obtain the integral equations for stresses and strain on the basis of a least-square approximation. The dual static constraint has been examined and new expressions provided to calculate the stress and strain invariants from microplane stresses and strains. The equivalence of the linear elastic microplane formulation to linear isotropic elasticity has also been established, along with the new conclusion that a double (simultaneously static and kinematic) constraint exists for a preferred choice of the elastic microplane moduli of the model with split.

The concepts of damage and plasticity may be systematically introduced in the microplane model, with interesting consequences on the resulting formulations. Microplane elasto-plasticity, with quite general assumptions, leads to a classical macroscopic elastoplastic formulation in which the plastic strains are obtained as an integral of the plastic strains on the microplanes. A simple example of a pressure-insensitive formulation has been proposed, which, with simple perfectly-plastic behavior on the microplanes, reproduces perfect von Mises plasticity as a limit case, with a natural hardening transition caused by progressive yielding of different microplanes.

Damage has been introduced in the microplanes in two forms. First, with different coefficients for the normal volumetric, deviatoric and tangential components of the microplane stresses, and second with a single damage coefficient per microplane affecting both the normal components (without split) and the tangential components. With appropriate assumptions, both forms lead to macroscopic expressions having the format of classical continuum damage mechanics with a fourth-order damage tensor and energy equivalence. In the first form, the damage tensor is obtained as an integral of the three damage coefficients 
for each microplane. This form may be considered a direct reformulation of the classical microplane model with kinematic constraint and volumetric-deviatoric split, exhibiting greater flexibility to incorporate new effects, but also the same limitations. The second form offers a new and apparently more attractive second-order damage tensor, which is obtained as an integral of the (single) microplane damage coefficient. This form may be related to previous formulations by other authors on damage theories based on second-order tensors.

Acknowledgements-Partial support from DGICYT (Madrid, Spain) under research grants PB92-0702 and PB930955 is gratefully acknowledged. The initial work of the second author was partly supported under US AFOSR grant No. 91-0140 to Northwestern University. In the final stage of the work, partial support was obtained under contract DACA 39-96-K-0049 between Waterways Experiment Station (WES), Vicksburg, Mississippi, and Northwestern University (monitored by Dr J. Zelasko). The authors wish also to thank Dr Pere C. Prat, for his valuable comments and suggestions.

\section{REFERENCES}

Batdorf, S. and Budiansky, B. (1949) A mathematical theory of plasticity based on the concept of slip. Technical Note 1871, National Advisory Committee for Aeronautics.

Bažant, Z. (1984) Microplane model for strain-controlled inelastic behavior. In Mechanics of Engineering Materials, eds C. Desai and R. Gallagher, pp, 45-59. John Wiley, London, U.K.

Bažant. Z. and Gambarova. P. (1984) Crack shear in concrete : crack band microplane model. ASCE. Journal of Structural Engineering 110, 2015-2035.

Bažant, Z., Jirásek, M., Xiang, Y., and Prat, P. (1994) Microplane model with stress-strain boundaries and its identification from tests with localized damage. In Computational Modelling of Concrete Structures, eds $\mathrm{H}$. Mang, N. Bićanić and R. de Borst ; pp. 255 261. Pineridge Press, Innsbruck.

Bažant, Z. and Oh, B. (1983) Microplane model for fracture analysis of concrete structures. In Proceedings of the Symposium on the Interaction of Non-Nuclear Munitions with Structures, pp. 49-55. U.S. Air Force Academy, Colorado Springs.

Bažant. Z. and Oh, B. (1985) Microplane model for progressive fracture of concrete and rock. ASCE Journal of Engineering Mechanics 111, 559-582.

Bažant, Z. and Prat, P. (1987) Creep of anisotropic clay: new microplane model. ASCE Journal of Engineering Mechanics 103(7), 1050-1064

Bažant, Z. and Prat, P. (1988a) Microplane model for brittle-plastic material: I. Theory. ASCE Journal of Engineering Mechanics 114(10), 1672-1688.

Bažant, Z. and Prat, P. (1988b) Microplane model for brittle-plastic material: II. Verification. ASCE Journal of Engineering Mechanics 114(10), 1689-1702.

Bažant, Z., Xiang, Y., and Prat, P. (1996a) Microplane model for concrete: I. Stress-strain boundaries and finite strain. ASCE Journal of Engineering Mechanics 122(3), 245-262.

Bažant, Z., Xiang, Y., Adley, M., Prat, P. and Akers, S. (1996b) Microplane model for concrete: II. Data delocalization and verification. ASCE Journal of Engineering Mechanics 122(3), 263268.

Carol, I. and Bažant, Z. (1991) Damage-rheology uncoupling for microplane damage tensor, with application to concrete with creep. In Constitutive Laws for Engineering Materials, eds C. Desai et al., pp. 391-394. ASME Press, Tucson, U.S.A.

Carol, I. and Bažant, Z. (1995) New developments in microplane/multicrack models for concrete. In Fracture Mechanics of Concrete Structures (FraMCoS 2), ed. F. Wittman, pp. 841-856. Aedificatio Publishers. Zürich, Switzerland

Carol, I., Bažant, Z and Prat, P. (1991) Geometric damage tensor based on microplane model. ASCE Journal of Engineering Mechanics 117, 2429-2448

Carol, I., Bažant, Z. and Prat, P. (1992a) Microplane-type constitutive models for distributed damage and localized cracking in concrete structures. In Fracture Mechanics of Concrete Structures (FraMCoS 1), ed. Z. Bažant, pp. 299-304. Elsevier, Breckenridge, U.S.A.

Carol, I.. Prat, P. and Bažant, Z. (1992b) New explicit microplane model for concrete: theoretical aspects and numerical implementation. International Journal of Solids and Structures 29(9), 1173-1191.

Carol, I., Rizzi, E. and Willam, K. (1994a) Towards a general formulation of elastic degradation and damage based on a loading surface. In Computational Modelling of Concrete Structures, eds H. Mang et al., pp. 199208. Pineridge Press, Innsbruck.

Carol, I., Rizzi, E. and Willam, K. (1994b) A unified theory of elastic degradation and damage based on a loading surface. International Journal of Solids and Structures 31(20), 2835-2865.

Carol, I. and Willam, K. (1996). Spurious energy dissipation/generation in modeling of stiffness and recovery for elastic degradation and damage. International Journal of Solids and Structures 33(20-22), 2939-2957.

Chow, J. and Wang, J. (1987) An anisotropic theory of continuum damage mechanics for ductile fracture. Engineering Fracture Mechanics 27, 547-558.

Cofer, W. (1992) Implementation of the nonlocal microplane concrete model within an explicit dynamic finite element program. Applied Mechanics Review 45(3), S132-S139.

Cofer, W. and Kohut, S. (1994) A general nonlocal microplane concrete material model for dynamic finite element analysis, Computers and Structures 53(1), 189-199.

Cordebois, J. and Sidoroff, F. (1982) Endommagement anisotrope en élasticité et plasticité. Journal de Mécanique Théorique et Appliquée Numero Spécial, 45-60.

Hasegawa, T. and Bažant, Z. (1993a) Nonlocal microplane concrete model with rate effect and load cycles. I: General formulation. ASCE Journal of Materials in Civil Engineering 5(3), 372-393. 
Hasegawa, T. and Bažant, Z. (1993b) Nonlocal microplane concrete model with rate effect and load cycles. II : Application and verification. ASCE Journal of Materials in Civil Engineering 5(3), 394-410.

Kachanov, L. (1958) Time rupture process under creep conditions. Izv. A. Rad. Nauk. SSSR otd Tekh. Nauk. 8: 26-31. (In Russian.)

Kachanov, M. (1980) Continuum model of medium with cracks. ASCE Journal of the Engineering Mechanics Division 106(EM5), 1039-1051.

Leckie, F. and Onat, E. (1981) Tensorial nature of damage measuring internal variables. In Proceedings, IUTAM Symposium on Physical Nonlinearities in Structural Analysis, eds J. Hult and J. Lemaitre, pp. 140-155. SpringerVerlag, Senlis, France.

Lemaitre, J. and Chaboche, J. (1985) Mécanique des materiaux solides. Dunod, Paris.

Lubarda, V. and Krajcinovic, D. (1993) Damage tensors and the crack density distribution. International Journal of Solids and Structures 30, 2859-2877.

Mohr, O. (1900) Welche Umstande bendingen der Bruch und der Elastizitätsgrenze des Materials. Z. Vereins Deutscher Ingenieure 44, 1-12.

Oda, M. (1983) A method for evaluating the effect of crack geometry on the mechanical behavior or cracked rock masses. Mechanics of Materials 2, 163-171.

Pande, G. and Sharma, K. (1983) Multilaminate model of clays-A numerical evaluation of the influence of rotation of principal axes. ASCE Journal of Engineering Mechanics 109(7), 397-418.

Prat, P. and Bažant, Z. (1989) Microplane model for triaxial deformation of soils. In Numerical Models in Geomechanics (NUMOG III), eds S. Pietruszczak, S. and G. Pande, pp. 139-146. Elsevier, Niagara Falls, Canada.

Prat, P. and Bažant, Z. (1991a) Microplane model for triaxial deformation of saturated cohesive soils. ASCE Journal of Geotechnical Engineering 117(6), 891-912.

Prat, P. and Bažant, Z. (1991b) A time-dependent microplane model for creep of cohesive soils. In Mechanics Computing in the 90 s and beyond, Proceedings of the ASCE-EMD Specialty Conference, ed H. Adeli and R. L. Sierakowski, pp. 1224-1228, ASCE. Columbus, Ohio, USA.

Rabotnov, Y. (1969) Creep Problems of Structural Members. North-Holland, Amsterdam.

Shen, W., Pen, L.-H., Yue, Y.-G., Shen, Z., and Tang, X.-D. (1989) Elastic damage and energy dissipation in anisotropic solid material. Engineering Fracture Mechanics 33, 273- 281.

Taylor, G. (1938) Plastic strain in metals. Journal of the Institute of Metals 62, 307-324.

Valanis, K. (1990) A theory of damage in brittle materials. Engineering Fracture Mechanics 36, 403-416.

Zienkiewicz, O. and Pande, G. (1977) Time-dependent multi-laminate model of rocks-A numerical study of deformation and failure of rock masses. International Journal of Numerical and Analytical Methods in Geomechanics 1, 219-247. 